\title{
On rank 2 vector bundles on Fano manifolds
}

\author{
Roberto Muñoz, Gianluca Occhetta, and Luis E. Solá Conde
}

\begin{abstract}
In this work we deal with vector bundles of rank two on a Fano manifold $X$ with second and fourth Betti numbers equal to one. We study the nef and pseudoeffective cones of the corresponding projectivizations and how these cones are related to the decomposability of the vector bundle. As consequences, we obtain the complete list of $\mathbb{P}^{1}$-bundles over $X$ that have a second $\mathbb{P}^{1}$-bundle structure, classify all the uniform rank two vector bundles on this class of Fano manifolds, and show the stability of indecomposable Fano bundles (with one exception on $\mathbb{P}^{2}$ ).
\end{abstract}

\section{Introduction}

While the classification of vector bundles on the complex projective line is a wellstated result, with numberless applications in algebraic geometry, the situation in higher dimensions is much more involved, even for vector bundles of low rank. For instance, no indecomposable rank two vector bundles on $\mathbb{P}^{n}, n \geq 5$, are known so far, despite of the efforts of many algebraic geometers interested in Hartshorne's conjecture (cf. [Ha1]).

On the other hand, several results in the literature provide splitting conditions for vector bundles on $\mathbb{P}^{n}$. In this direction, the work of Ancona, Peternell and Wiśniewski (cf. [APW]) is of particular interest for our purposes: they show, using techniques of Mori theory, that if a rank two vector bundle $\mathcal{E}$ is indecomposable, then $-K_{\mathbb{P}(\mathcal{E})}$ cannot be ample, with the exception of a precise list of bundles.

In a previous paper (cf. [MOS1]) we showed that the amplitude of $-K_{\mathbb{P}(\mathcal{E})}$ might be replaced by milder positivity conditions. Here we address the problem of understanding how splitting may be inferred from positivity, in the more general setting of rank two vector bundles on Fano manifolds of Picard number one. More concretely, we study the nef and pseudoeffective cones of $\mathbb{P}(\mathcal{E})$. They are completely determined by their slopes $\tau$ and $\rho$ (see Definition 2.1), and we show that their values are tightly related with the (in)decomposability of the bundle (see, e.g., Corollary 5.9).

Kyoto Journal of Mathematics, Vol. 54, No. 1 (2014), 167-197

DOI 10.1215/21562261-2400310, (C) 2014 by Kyoto University

Received June 14, 2012. Revised November 16, 2012. Accepted June 15, 2013.

2010 Mathematics Subject Classification: Primary 14M15; Secondary 14E30, 14J45.

Muñoz's and Solá Conde's work partially supported by Spanish government project MTM2009-06964.

Occhetta's work partially supported by Ministero Istruzione Università Ricerca (PRIN project: Proprietà geometriche delle varietà reali e complesse). 
The structure of the paper is the following: in Sections 2 and 4 we bound the set of values of the pair $(\tau, \rho)$ for an indecomposable vector bundle under different sets of hypotheses. We also include a number of examples in which these invariants are computed and find relations with the Nagata conjecture for plane curves (see Example 2.13).

In Section 3 we pay special attention to the behavior of these cones with respect to rational curves contained in $X$ and with the loci of minimal sections on them: it is in fact a fundamental question whether these cones are determined by rational curves in $\mathbb{P}(\mathcal{E})$.

The second fundamental ingredient of our work, with which we deal in Section 5, is a splitting criterion based on [APW, Theorem 10.5] and [B, Theorem 1]. It basically says (see Corollaries 5.5, 5.6 for details), that if $\mathcal{E}$ is indecomposable, then RatCurves $(\mathbb{P}(\mathcal{E}))_{y}$ cannot contain complete curves for the general $y \in \mathbb{P}(\mathcal{E})$. As a consequence we obtain that $\mathcal{E}$ decomposes unless $\tau$ is sufficiently large.

Throughout Sections 4 and 5 we will also see how our arguments get enhanced if we make some further assumptions on the cones of $\mathbb{P}(\mathcal{E})$, semiampleness of the generators of $\operatorname{Nef}(X)$, for instance. In this last case (see Proposition 4.12), applying some results of number theory to our formulas, we obtain severe restrictions on the invariants of $(X, \mathcal{E})$.

On the other hand, assuming that $\mathcal{E}$ is Fano (i.e., $\mathbb{P}(\mathcal{E})$ Fano) allows us to put together the Mori machinery and our tools. This leads in Theorem 6.3 to a proof of a Grauert-Schneider-type result: indecomposable rank two Fano bundles on Fano manifolds of Picard number one and fourth Betti number $b_{4}(X)=1$ are stable (with one exception on $\mathbb{P}^{2}$ ). Furthermore, under these hypotheses, we give, in Theorem 6.5, the complete list of Fano bundles satisfying that $i_{X}-$ $c_{1}$ is even, where $i_{X}$ is the index of $X$ and $c_{1}$ is an integer corresponding to the first Chern class of $\mathcal{E}$ as explained in Section 1.1. These bundles are those whose projectivization has a second $\mathbb{P}^{1}$-bundle structure. This results leads, in particular, to a complete classification of uniform rank two vector bundles on Fano manifolds under some assumptions (see Corollary 6.7).

The techniques and results of the present paper can be applied also to classify Fano bundles satisfying that $i_{X}-c_{1}$ is odd. The proof in this case is longer and more complicated than the one of Theorem 6.5, so we will present it in another paper [MOS2].

Finally, in Section 7 we show how our techniques may be used to improve some well-known results concerning Hartshorne's conjecture for codimension two subvarieties of $\mathbb{P}^{n}$.

\subsection{Setup}

Throughout this paper we will work in the following general setup.

SETUP 1.1

$X$ will be a complex Fano manifold of dimension $n$ whose Picard group is generated by the ample line bundle $\mathcal{O}_{X}\left(H_{X}\right)$, and $\mathcal{E}$ will be a normalized rank two 
vector bundle on $X$, that is, whose determinant equals $\mathcal{O}_{X}\left(c_{1} H_{X}\right)$, with $c_{1}=0$ or -1 .

Using the isomorphism $\operatorname{Pic}(X) \cong \mathbb{Z}\left\langle H_{X}\right\rangle$, we will freely identify a line bundle with the corresponding integer. In particular, the determinant of $\mathcal{E}$ and the anticanonical line bundle are identified with integers $c_{1}$ and $i_{X}$ (index of $X$ ). If $a$ is an integer and $\mathcal{F}$ is a sheaf on $X, \mathcal{F}(a)$ denotes the sheaf $\mathcal{F} \otimes \mathcal{O}_{X}\left(a H_{X}\right)$.

As usual $\mathbb{P}(\mathcal{E})$ denotes the Grothendieck projectivization of $\mathcal{E}$, that is, $\mathbb{P}(\mathcal{E})=$ $\operatorname{Proj}_{X}\left(\bigoplus_{k>0} S^{k} \mathcal{E}\right)$. The tautological line bundle on $\mathbb{P}(\mathcal{E})$ will be denoted by $\mathcal{O}(1)$, the natural projection from $\mathbb{P}(\mathcal{E})$ to $X$ by $\pi$, and the pullback of $H_{X}$ to $\mathbb{P}(\mathcal{E})$ by $H$. By $L$ we will denote a divisor with associated line bundle $\mathcal{O}(1)$, and by $-K_{\text {rel }}$ a relative canonical divisor of $\mathbb{P}(\mathcal{E})$ over $X$, that is, a divisor associated to the line bundle $\operatorname{det}\left(\pi^{*} \mathcal{E}^{\vee}(1)\right)$. The second Chern class of $\mathcal{E}$ will be denoted by $c_{2}(\mathcal{E}) \in H^{4}(X, \mathbb{Z})$, and its discriminant $c_{1}^{2}(\mathcal{E})-4 c_{2}(\mathcal{E})$ by $\Delta(\mathcal{E})$.

The Mori cone of $\mathbb{P}(\mathcal{E})$ will be denoted by $\overline{\operatorname{NE}(\mathbb{P}(\mathcal{E}))}$. It has two extremal rays, $R_{1}$ and $R_{2}$, where $R_{1}$ corresponds to $\pi$. The ray $R_{2}$ will often be referred to as the second extremal ray of $\mathbb{P}(\mathcal{E})$. The dual cone of $\overline{\mathrm{NE}(\mathbb{P}(\mathcal{E}))}$ is the nef cone of $\mathbb{P}(\mathcal{E})$, denoted by $\operatorname{Nef}(\mathbb{P}(\mathcal{E}))$. We will also consider the pseudoeffective cone of $\mathbb{P}(\mathcal{E})$, denoted by $\overline{\operatorname{Eff}(\mathbb{P}(\mathcal{E}))}$ : the closure of the convex cone generated by effective divisors. By $[\mathrm{BDPP}]$, it is the dual of the cone of movable curves $\overline{\operatorname{ME}(\mathbb{P}(\mathcal{E}))}$.

Some of our results will require intersection theory of cycles of codimension one and two. When doing this we will abuse of notation and freely identify cycles with their numerical classes. We will sometimes assume that $b_{4}(X)=1$, in which case the quotient of $H^{4}(X, \mathbb{Z})$ modulo numerical equivalence is isomorphic to $\mathbb{Z}$. We will denote by $\Sigma$ a positive (meaning that $\Sigma \cdot H^{n-2}>0$ ) generator $\Sigma$ of this group, so that we may write $c_{2}(\mathcal{E})=c_{2} \Sigma, H_{X}^{2}=d \Sigma, \Delta(\mathcal{E})=\left(d c_{1}^{2}-4 c_{2}\right) \Sigma=: d \Delta \Sigma$ for some $c_{2}, d \in \mathbb{Z}$. In particular the usual Chern-Wu relation on $\mathbb{P}(\mathcal{E})$ may be written as $K_{\text {rel }}^{2}=\Delta H^{2}$.

We will sometimes consider the minimum integer $\beta$ such that $\mathcal{E}(\beta)$ has nonzero global sections, so that there is an exact sequence

$$
0 \rightarrow \mathcal{O}_{X}(-\beta) \longrightarrow \mathcal{E} \longrightarrow \mathcal{I}_{Z}\left(c_{1}+\beta\right) \rightarrow 0,
$$

where $Z \subset X$ has pure codimension two and its cohomology class is $c_{2}(\mathcal{E}(\beta))$. The interest of this sequence relies on the fact that, since $X$ is Fano, the bundle $\mathcal{E}$ decomposes as a sum of line bundles if and only if $Z=\emptyset$. This is in fact equivalent to $Z$ being numerically equivalent to 0 , since $Z \cdot H^{n-2}>0$ when $Z$ is nonempty.

By definition, $\mathcal{E}$ is stable (resp., semistable) if $\beta>-c_{1} / 2$ (resp., $\beta \geq-c_{1} / 2$ ). Recall that, by Bogomolov inequality and the Mehta-Ramanathan theorem, if $\mathcal{E}$ is semistable, then $\Delta(\mathcal{E}) \cdot H_{X}^{n-2} \leq 0$. See [HL] for a complete account on stability of sheaves. If $X=\mathbb{P}^{n}$, it is well known that $\mathcal{E}$ is not stable if $\Delta=0$ (see [Bt, Corollary 1]). We will prove in Lemma 2.5 that a similar statement holds for Fano manifolds of Picard number one.

Finally, we will consider rational curves in $X$, for which we will adopt the notation and conventions appearing in $[\mathrm{K}]$. Given a rational curve $\ell \subset X$, the 
pullback of $\mathcal{E}$ via the normalization of $\ell$ takes the form $\mathcal{O}_{\mathbb{P}^{1}}(a) \oplus \mathcal{O}_{\mathbb{P}^{1}}(b)$ with $a+b=\left(H_{X} \cdot \ell\right) c_{1}$. We will say that $\ell$ has splitting type $(a, b)$ with respect to $\mathcal{E}$, or that $\mathcal{E}$ has splitting type $(a, b)$ with respect to $\ell$. A rational curve of $H_{X^{-}}$ degree one is called a line. Given a family of rational curves $\mathcal{M}$ in $X$ (i.e., a component of RatCurves $\left.{ }^{n}(X)\right)$ of $H_{X}$-degree $\mu$, with universal family $p: \mathcal{U} \rightarrow \mathcal{M}$ and evaluation morphism ev $: \mathcal{U} \rightarrow X$, and given a nonnegative integer $t$, we will denote by $\mathcal{M}^{t} \subset \mathcal{M}$ the locally closed subset, endowed with the reduced scheme structure, parameterizing curves on which $\mathcal{E}$ has splitting type $\left(\left(c_{1}+t\right) \mu / 2,\left(c_{1}-\right.\right.$ t) $\mu / 2)$. In the same way we will use $p^{t}: \mathcal{U}^{t} \rightarrow \mathcal{M}^{t}$ and $\mathrm{ev}^{t}: \mathcal{U}^{t} \rightarrow X$ to denote the corresponding associated maps. We say that $\mathcal{E}$ is uniform with respect to $\mathcal{M}$ if $\mathcal{M}=\mathcal{M}^{t}$ for some $t$.

Given an element $\ell \in \mathcal{M}^{t}$ we will sometimes consider minimal sections of $\mathbb{P}(\mathcal{E})$ over $\ell$ that will be denoted by $\tilde{\ell}$. The set of minimal sections over curves of $\mathcal{M}^{t}$ is a family of rational curves $\operatorname{in} \mathbb{P}(\mathcal{E})$ that we will denote by $\widetilde{\mathcal{M}}^{t}$. We will use $\widetilde{p}^{t}$ and $\widetilde{\mathrm{ev}}^{t}$ to denote the corresponding morphisms.

As usual, a subindex ()$_{x}$ on $\mathcal{M}, \mathcal{M}^{t}, \widetilde{\mathcal{M}}^{t}$, and so on, means that we are restricting ourselves to curves passing through the point $x$.

Given a proper family $\mathcal{M}$ of rational curves or of rational 1-cycles, and a point $x \in \operatorname{Locus}(\mathcal{M})$ we will denote by $\operatorname{ChLocus}_{x}(\mathcal{M})$ the equivalence class of $x$ with respect to the set-theoretic relation associated to the proper proalgebraic relation Chain $(\mathcal{U})$ (cf. [K, IV.4.8]). It is the closed subset of $X$ consisting of points that can be joined to $x$ by a connected chain of cycles parameterized by $\mathcal{M}$.

We will use the fact that the numerical class of every curve in $\operatorname{ChLocus}_{x}(\mathcal{M})$ can be written as a linear combination of the numerical classes of irreducible components of cycles parameterized by $\mathcal{M}$.

\section{The nef cone of a vector bundle}

It is well known that the relative anticanonical divisor of a smooth nonconstant surjective morphism between smooth projective varieties is not ample (cf. [KMM, Corollary 2.8]). In the next definition we introduce two invariants that give a measure of the (lack of) positivity of $-K_{\text {rel }}$. For simplicity we will stick to Setup 1.1, though the definitions are meaningful in a much broader setting.

\section{DEFINITION 2.1}

Given $(X, \mathcal{E})$ as in Setup 1.1 we denote by $\tau(\mathcal{E})$ the only real number such that $-K_{\text {rel }}+\tau H$ is nef but not ample, and we call it the slope of $\operatorname{Nef}(\mathbb{P}(\mathcal{E}))$. In a similar manner, we define $\rho(\mathcal{E})$ as the only real number such that $-K_{\text {rel }}+\rho H$ is pseudoeffective but not big, and call it the slope of $\overline{\operatorname{Eff}(\mathbb{P}(\mathcal{E}))}$. (We refer the interested reader to $[\mathrm{L}, 2.2 \mathrm{~B}$ ] for details on pseudoeffective divisors.) Equivalently, we may have defined

$$
\begin{aligned}
& \tau(\mathcal{E})=\sup \{\tau(\mathcal{E}, C) \mid C \text { irreducible curve in } \mathbb{P}(\mathcal{E})\} \\
& \rho(\mathcal{E})=\sup \{\tau(\mathcal{E}, C) \mid C \text { irreducible movable curve in } \mathbb{P}(\mathcal{E})\},
\end{aligned}
$$


where

$$
\tau(\mathcal{E}, C):= \begin{cases}-\infty & \text { if } C \text { is a fiber of } \pi, \\ \frac{K_{\mathrm{rel}} \cdot C}{H \cdot C} & \text { otherwise. }\end{cases}
$$

If there is no possible confusion we will use $\tau, \rho$, and $\tau(C)$ instead of $\tau(\mathcal{E})$ and $\tau(\mathcal{E}, C)$.

\section{REMARK 2.2}

If $\ell$ is a rational curve of splitting type $(a, b)$ with respect to $\mathcal{E}$ and $\tilde{\ell}$ is a minimal section of $\mathbb{P}\left(\mathcal{E}_{\mid \ell}\right)$ over $\ell$, then $\tau(\widetilde{\ell})=|b-a| / H_{X} \cdot C \geq 0$. Since it depends only on $\mathcal{E}_{\mid \ell}$ and $\ell$, abusing notation, we will usually write $\tau(\ell)$ instead of $\tau(\widetilde{\ell})$. In particular, with the notation introduced in Section 1.1, we have that $\ell \in \mathcal{M}^{t}$ iff $\tau(\ell)=t$.

Throughout the rest of this section we will discuss some features of $\tau$ and the nef cone of $\mathbb{P}(\mathcal{E})$. In the following theorem we show that the lowest value of $\tau$ is only achieved by the trivial bundle. Note that the same proof works for vector bundles of any rank.

\section{THEOREM 2.3}

Let $(X, \mathcal{E})$ be as in Setup 1.1. Then $\tau \geq 0$, and equality holds if and only if $\mathcal{E} \cong \mathcal{O}_{X}^{\oplus 2}$.

Proof

The first assertion follows by definition of $\tau$ and the fact that the $\mathbb{Q}$-twisted bundle $\mathcal{E}\left(-c_{1} / 2\right)$ is not ample since its determinant is zero.

If $\tau=0$, then Remark 2.2 tells us that the splitting type of every rational curve $\ell$ with respect to $\mathcal{E}$ is either $(0,0)$ or $\left(-H_{X} \cdot \ell / 2,-H_{X} \cdot \ell / 2\right)$. Then the conclusion follows from the proposition below (cf. also [BdS, Theorem 2.2], where the same result is proved with different techniques).

\section{PROPOSITION 2.4}

Let $M$ be a rationally connected manifold, and let $\mathcal{F}$ be a rank $r$ vector bundle verifying that $\mathbb{P}\left(\mathcal{F}_{\mid \ell}\right) \cong \ell \times \mathbb{P}^{r-1}$ for every rational curve in $M$. Then $\mathbb{P}(\mathcal{F}) \cong$ $M \times \mathbb{P}^{r-1}$ is trivial.

Proof

Let us denote by $\pi: \mathbb{P}(\mathcal{F}) \rightarrow M$ the canonical projection. Take a component $\mathcal{C}$ of Chow $(M)$ containing the class a very free (see [D, Definition 4.5]) rational curve $C \subset M$, and let $\widetilde{\mathcal{C}}$ be the component of $\operatorname{Chow}(\mathbb{P}(\mathcal{F}))$ containing the class of a minimal section $\widetilde{C}$ of $\mathbb{P}(\mathcal{F})$ over $C$. By construction $\widetilde{\mathcal{C}}$ dominates $\mathbb{P}(\mathcal{F})$ and we may consider the quotient $\varphi: \mathbb{P}(\mathcal{F}) \rightarrow Y$ associated to the proper algebraic relation given by $\widetilde{\mathcal{C}}$. 
We claim first that $\operatorname{dim} Y \leq r-1$. In fact, take two general points $x_{1}, x_{2} \in M$. There exists an irreducible curve $C^{\prime}$ of $\mathcal{C}$ joining $x_{1}$ and $x_{2}$; hence, given any element $y_{1} \in \pi^{-1}\left(x_{1}\right)$, we may choose a section of $\mathbb{P}(\mathcal{F})$ over $C^{\prime}$ meeting $y_{1}$ and $\pi^{-1}\left(x_{2}\right)$. Then Chlocus $(\widetilde{\mathcal{C}})_{y_{1}}$ intersects the general fiber of $\pi$; hence its dimension is bigger than or equal to $\operatorname{dim} M$. Since $\varphi^{-1}\left(\varphi\left(y_{1}\right)\right)$ contains $\operatorname{Chlocus}(\widetilde{\mathcal{C}})_{y_{1}}$, the claim follows.

Let $L$ and $D$ be divisors in $\mathbb{P}(\mathcal{F})$ associated to $\mathcal{O}_{\mathbb{P}(\mathcal{F})}(1)$ and $\pi^{*}(\operatorname{det} \mathcal{F})$, respectively, and consider the $\mathbb{Q}$-divisor $L-D / r$ which, by construction, has intersection zero with every cycle of $\widetilde{\mathcal{C}}$ and positive intersection with curves on fibers of $\pi$. Furthermore, it is nef on rational curves in $\mathbb{P}(\mathcal{E})$. In fact, given a (nonvertical) rational curve $\ell_{0} \subset \mathbb{P}(\mathcal{F})$, we may consider the normalization $\ell$ of its projection into $M$; since $\mathcal{F}_{\mid \ell} \cong \mathcal{O}_{\mathbb{P}^{1}}\left(D \cdot \ell_{0} / r\right)^{\oplus r}$, it follows that $L-D / r$ is nef on $\mathbb{P}\left(\mathcal{F}_{\mid \ell}\right)$ and thus on $\ell_{0}$.

In particular $L-D / r$ has intersection zero with every component of a cycle of $\widetilde{\mathcal{C}}$. If $\operatorname{dim} Y$ were strictly smaller than $r-1$, the general fiber of $\varphi$ would intersect the fibers of $\pi$ in positive dimension, but this is impossible, since $L-$ $D / r$ has positive intersection with curves contained in the fibers of $\pi$. Therefore $\operatorname{dim} Y=r-1$.

Denoting by $Z$ a general fiber of $\varphi$ and by $p: Z \rightarrow M$ the natural projection we may consider the projective bundle $\mathbb{P}\left(p^{*} \mathcal{F}\right)$. It admits a section whose image we denote by $Z^{\prime}$, and we have a commutative diagram:

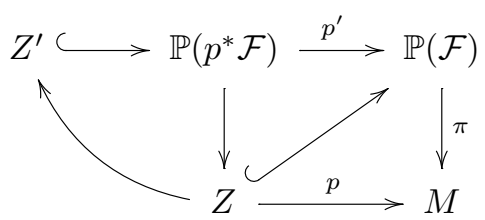

Since $-K_{\mathbb{P}(\mathcal{F})}=-\pi^{*} K_{M}+r L-D$ we have $-\left.K_{Z} \equiv\left(-\pi^{*} K_{M}\right)\right|_{Z}$; hence $-K_{Z}=-\left(\left.\pi\right|_{Z}\right)^{*} K_{M}$. This implies that $Z$ is a section of $\pi$, since $M$ is simply connected.

Finally, we consider the surjective morphism $\mathcal{F} \rightarrow \mathcal{F}^{\prime \prime}$ determined by the section $Z$ and denote by $\mathcal{F}^{\prime}$ its kernel. With this notation, the normal bundle $N_{Z}$ of $Z$ in $\mathbb{P}(\mathcal{F})$ is isomorphic to $\mathcal{F}^{\prime \vee} \otimes \mathcal{F}^{\prime \prime}$. On the other hand we know that $N_{Z}$ is trivial; therefore $\mathcal{F}^{\prime} \cong \mathcal{F}^{\prime \prime \oplus r-1}$. Finally, the rational connectedness of $M$ provides $H^{1}\left(M, \mathcal{O}_{M}\right)=0$ and the splitting of the sequence

$$
0 \rightarrow \mathcal{F}^{\prime \prime \oplus r-1} \longrightarrow \mathcal{F} \longrightarrow \mathcal{F}^{\prime \prime} \rightarrow 0 \text {. }
$$

As a consequence of Proposition 2.4 we obtain the following result on the stability of $\mathcal{E}$ when $\Delta=0$, which will be used in Section 5 to make our splitting criteria for vector bundles work also in the case $\Delta=0$ (see Lemma 5.4).

\section{LEMMA 2.5}

Let $(X, \mathcal{E})$ be as in Setup 1.1 with $\Delta=0$. Then $\mathcal{E}$ is not semistable unless $\mathcal{E}$ is trivial. 
Proof

Let us assume first that $c_{1}=0$. By [MR2, Theorem. 5.1] no stable vector bundle of rank $\geq 2$ with $c_{1}=c_{2}(\mathcal{E}) \cdot H^{n-2}=0$ on $X$ exists, since $X$ is simply connected. If $\mathcal{E}$ is semistable, then $h^{0}(\mathcal{E}) \neq 0$ and $h^{0}(\mathcal{E}(-1))=0$, so that $\mathcal{E}$ is trivial, as $c_{2}(\mathcal{E})$ is numerically trivial.

If $c_{1}=-1$ and $\mathcal{E}$ is semistable (hence stable) then $S^{2} \mathcal{E}(1)$ is a direct sum of stable vector bundles whose first Chern class is zero (cf. [HL, Theorem 3.2.11]). Since its rank is three, the second Chern class of every direct summand is zero, too. Applying [MR2, Theorem 5.1] to each summand, we get that $S^{2} \mathcal{E}(1)$ is trivial. Hence, for any rational curve $\ell \subset X$, we have $\mathcal{E}_{\mid \ell}=\mathcal{O}\left(-H_{X} \cdot \ell / 2,-H_{X}\right.$. $\ell / 2$ ), contradicting Proposition 2.4.

We will often consider the restriction of $\mathcal{E}$ to curves in $X$. Let us then recall some well-known facts about ruled surfaces (we refer the reader to [Ha2, V Section 2] for details).

\section{REMARK 2.6}

Given a smooth curve $C$ and a $\mathbb{P}^{1}$-bundle $\mathbb{P}(\mathcal{F})$, the vector space $N^{1}(\mathbb{P}(\mathcal{F}))$ is generated by the class of a minimal section $\widetilde{C}$ and the class of a fiber $F$. Recall that $\mathcal{F}$ is stable (resp., semistable) if and only if the self-intersection $-e$ of $\widetilde{C}$ is positive (resp., nonnegative). If $\mathcal{F}$ is not semistable, then the nef cone of $\mathbb{P}(\mathcal{F})$ is generated by the classes of $F$ and $\widetilde{C}+e F$ and the pseudoeffective cone by the classes of $F$ and $\widetilde{C}$; moreover, the only irreducible curve whose class does not lie in the nef cone is $\widetilde{C}$. If $\mathcal{F}$ is semistable, then the two cones coincide, and they are generated by the classes of $F$ and $2 \widetilde{C}+e F$.

Given any curve in $X$ we may consider the restriction of $\mathcal{E}$ to its normalization $C$, which we denote by $\mathcal{E}_{\mid C}$. Given any irreducible curve $D$ in $\mathbb{P}(\mathcal{E})$ we may consider the normalization $\iota: C \rightarrow X$ of $\pi(D)$ and the strict transform $D^{\prime}$ of $D$ in $\mathbb{P}\left(\mathcal{E}_{\mid C}\right)$, obtaining

$$
\tau(D)=\frac{K_{\mathrm{rel}} \cdot D}{H \cdot D}=\frac{K_{\mathbb{P}\left(\mathcal{E}_{\mid C}\right) \mid C \cdot D^{\prime}}}{\iota^{*} H \cdot D^{\prime}} \leq \tau\left(C_{1}\right),
$$

where $C_{1}$ is an effective 1-cycle defined as the pushforward into $\mathbb{P}(\mathcal{E})$ of $\widetilde{C}$ if $\mathcal{E}_{\mid C}$ is not semistable, and of $2 \widetilde{C}+e F$ otherwise. Note that $K_{\mathbb{P}\left(\mathcal{E}_{\mid C}\right) \mid C} \cdot \widetilde{C}=e$ and $K_{\mathbb{P}\left(\mathcal{E}_{\mid C}\right) \mid C} \cdot(2 \widetilde{C}+e F)=0$; hence $\tau\left(C_{1}\right)$ is bigger than 0 if $\mathcal{E}_{\mid C}$ is not semistable and 0 otherwise.

In particular, we may state the following.

\section{LEMMA 2.7}

Let $(X, \mathcal{E})$ be as in Setup 1.1, and assume that $\mathcal{E}$ is not trivial. Then

$$
\tau=\sup \left\{\tau(\widetilde{C}) \mid \mathcal{E}_{\mid C} \text { not semistable }\right\} .
$$


Proof

By the previous discussion, we only need to show that there exists a curve in $X$ on which $\mathcal{E}$ is not semistable. If this were not the case, then $\tau(\ell)$ would be zero for every rational curve $\ell$ in $X$ and $\mathcal{E}$ would be trivial by Proposition 2.4.

\section{REMARK 2.8}

A central question on the theory of vector bundles on Fano manifolds is to which extent the geometry of the bundle is determined by its behavior on rational curves. Proposition 2.4 and Lemma 2.7 motivate us to define

$$
\tau_{0}:=\sup \{\tau(C) \mid C \text { rational }\} .
$$

So far, we do not know of any example of rank two vector bundles for which $\tau \neq \tau_{0}$.

\section{REMARK 2.9}

It is well known that a vector bundle with $\tau<i_{X}$ (known in the literature as a Fano bundle, cf. [APW]) satisfies the following properties:

- (Rationality) $\tau \in \mathbb{Q}$;

- (Base point freeness $)-K_{\text {rel }}+\tau H$ is semiample;

- (Rational curves in the second ray) there exists a rational curve $\ell$ for which $\ell \cdot\left(-K_{\text {rel }}+\tau H\right)=0$. In particular $\tau=\tau_{0}$.

It is then natural to ask whether they are satisfied in broader classes of vector bundles on Fano varieties. Section 2.1 below contains a number of examples in which we deal with this question.

\subsection{Examples}

EXAMPLE 2.10

If $\mathcal{E} \cong \mathcal{O}_{X}(a) \oplus \mathcal{O}_{X}(b)$, then $-K_{\text {rel }}+|b-a| H$ is semiample but not ample, and the corresponding morphism contracts a section of $\mathbb{P}(\mathcal{E})$ over $X$, containing the minimal sections of $\mathcal{E}$ over rational curves in $X$. Note that this in particular shows that $\tau$ is not upper bounded in the class of vector bundles on $X$.

\section{EXAMPLE 2.11}

Indecomposable Fano bundles on projective spaces and quadrics have been extensively studied and completely classified. We refer the interested reader to [SW2], [SW1], and $[\mathrm{APW}]$. It is well known that the second contraction of the corresponding projectivization is:

- either a smooth blow-up (for $X=\mathbb{P}^{2}$ or $\mathbb{Q}^{4}$ ), or

- a conic bundle (for $X=\mathbb{P}^{2}$ or $\mathbb{Q}^{3}$ ), or

- a $\mathbb{P}^{1}$-bundle (for $X=\mathbb{P}^{2}, \mathbb{P}^{3}, \mathbb{Q}^{3}$ or $\mathbb{Q}^{5}$ ), or

- a $\mathbb{P}^{2}$-bundle (for $\left.\mathbb{Q}^{4}\right)$. 
A straightforward computation for every example of the list shows that $\tau=i_{X}-1$ unless for $\mathbb{P}^{d}$ bundles, for which $\tau$ equals $i_{X}-(d+1)$.

There are examples of non-Fano bundles for which $\mathbb{P}(\mathcal{E})$ enjoys the properties stated in Remark 2.9. That is the case, for instance, of the Horrocks-Mumford bundle.

\section{EXAMPLE 2.12}

Let $\mathcal{F}_{H M}$ denote the Horrocks-Mumford bundle on $\mathbb{P}^{4}$. The possible splitting types of lines with respects to $\mathcal{F}_{H M}$ are $(2,3),(1,4),(0,5)$, and $(-1,6)$ (cf. [HM]); hence $7 \leq \tau_{0} \leq \tau$. On the other hand the bundle $\mathcal{F}_{H M}(1)$ is globally generated (cf. [Su, Proposition 5]); therefore $\tau=7$, and the second ray of the Mori cone is generated by the class of a section of $\mathbb{P}\left(\mathcal{F}_{H M}\right)$ over a line of splitting type $(-1,6)$.

Finally we include here an example on $\mathbb{P}^{2}$ due to Schwarzenberger (see [OSS, Theorem 2.2.5] for details). We will see that one should not expect $\tau$ to be rational in general. Furthermore, even if $\tau$ is rational, there could be no rational classes in the second extremal ray of $\overline{\operatorname{NE}(\mathbb{P}(\mathcal{E}))}$.

\section{EXAMPLE 2.13 ([OSS, THEOREM. 2.2.5])}

Consider a finite set $\mathcal{P}=\left\{P_{1}, \ldots, P_{k}\right\} \subset \mathbb{P}^{2}$ of points in the complex projective plane. Blow up $\mathbb{P}^{2}$ along $\mathcal{P}$ to get $\sigma: \mathbb{B} \rightarrow \mathbb{P}^{2}$, and denote by $E=E_{1}+\cdots+E_{k}$ the exceptional divisor. The Schwarzenberger bundle maybe defined as the only bundle $\mathcal{E}$ whose pullback to $\mathbb{B}$ is an extension of $\mathcal{O}_{\mathbb{B}}(-E)$ by $\mathcal{O}_{\mathbb{B}}(E)$, whose restriction to $E$ is the standard Euler sequence on $E$.

Now observe that the nefness of $\mathcal{E}(\tau / 2)$ is equivalent to that of its pullback via $\sigma$. Then $\mathcal{E}(\tau / 2)$ is nef if and only if $\sigma^{*} H-(2 / \tau) E$ is nef and, in particular,

$$
\frac{2}{\tau}=\epsilon\left(\mathcal{O}_{\mathbb{P}^{2}}(1) ; \mathcal{P}\right)
$$

where $\epsilon\left(\mathcal{O}_{\mathbb{P}^{2}}(1) ; \mathcal{P}\right)$ denotes the Seshadri constant of the line bundle $\mathcal{O}_{\mathbb{P}^{2}}(1)$ with respect to the set of $k$ points $\mathcal{P}$. In particular, if $k \geq 9$ one gets $\tau \geq 2 k^{1 / 2}$ (see, for instance [Ba, Section 8]), and the famous Nagata conjecture may be rephrased as follows.

\section{CONJECTURE 2.14 (NAGATA)}

With the same notation as above, if $\mathcal{P} \subset \mathbb{P}^{2}$ is very general and $k \geq 9$, then $\tau=2 k^{1 / 2}$.

A proof of this would provide an example in which $\tau$ is not rational, but this conjecture has been proven only when $k^{1 / 2}$ is an integer (cf. [N]). Note that from Nagata's proof it follows that $\mathcal{E}\left(k^{1 / 2}\right)$ is ample on every curve in $\mathbb{P}^{2}$ if $k^{1 / 2}$ is an integer bigger than or equal to 4 . In particular, in this case the exact value of $\tau$ is not achieved on any particular curve in $\mathbb{P}^{2}$ and $-K_{\text {rel }}+\tau H$ is not semiample. 
Let us focus now on the situation in which $\mathcal{P}$ consists of 9 very general points. In this case $\tau=6$ and $\mathcal{E}(3)$ is nef and not ample on the (unique) smooth elliptic cubic curve $C$ containing $\mathcal{P}$. It is well known that there are no rational curves in $\mathbb{B}$ numerically proportional to the strict transform of $C$; hence $\tau(\ell) \neq 6$ for every rational curve $\ell$.

On the other hand, let $\psi: \mathbb{P}^{2} \rightarrow \mathbb{P}^{2}$ be the composition of three consecutive Cremona transformations, based on $P_{1}, P_{2}, P_{3}$, then on $P_{4}, P_{5}, P_{6}$, and finally on $P_{7}, P_{8}, P_{9}$. Starting with the line $\ell_{0}$ by $P_{8}$ and $P_{9}$, consider the sequence of rational curves defined by $\ell_{n+1}:=\psi_{*} \ell_{n}$. A direct computation provides $\tau\left(\ell_{n}\right)=$ $6-16 /\left((-1)^{n}+18 n^{2}+24 n+7\right)$. Hence we get an example in which $\tau=\tau_{0}$ but is not computed by a rational curve.

\section{Stability of $\mathcal{E}$ versus pseudoeffective divisors of $\mathbb{P}(\mathcal{E})$}

In this section we study the relation between the slope $\rho$ of $\overline{\operatorname{Eff}(\mathbb{P}(\mathcal{E}))}$ and the stability of $\mathcal{E}$. We include some preliminary results on families of minimal sections of $\mathbb{P}(\mathcal{E})$ over rational curves that will be useful here and in the forthcoming sections. Recall that

$$
\rho=\sup \{\tau(D) \mid D \text { irred. movable curve in } \mathbb{P}(\mathcal{E})\} .
$$

Given a curve in $X$ with normalization $\iota: C \rightarrow X$ such that $\mathcal{E}_{\mid C}=\iota^{*} \mathcal{E}$ is not semistable, we will denote by $\widetilde{C}$ the image into $\mathbb{P}(\mathcal{E})$ of the minimal section of $\mathbb{P}\left(\mathcal{E}_{\mid C}\right)$ over $C$. Then, arguing as in Remark 2.6, we may prove the following.

\section{LEMMA 3.1}

Let $(X, \mathcal{E})$ be as in Setup 1.1, and assume that $\mathcal{E}$ is semistable. Then

$$
\rho=\sup \left(\{0\} \cup\left\{\tau(\widetilde{C}) \mid \widetilde{C} \text { movable in } \mathbb{P}(\mathcal{E}), \mathcal{E}_{\mid C} \text { not semistable }\right\}\right) \geq 0 .
$$

\section{Proof}

We begin by showing that $\rho \geq 0$; in fact, given $\delta<0$, we will find a movable curve $C_{\delta} \subset \mathbb{P}(\mathcal{E})$ satisfying $\tau\left(C_{\delta}\right) \geq \delta$. In order to see this, fix a general complete intersection curve $C$ in $X$, and consider the restriction $\mathcal{E}_{\mid C}$, which is semistable by the Mehta-Ramanathan restriction theorem. The nef cone of $\mathbb{P}\left(\mathcal{E}_{\mid C}\right)$, which is equal to $\overline{\operatorname{ME}\left(\mathbb{P}\left(\mathcal{E}_{\mid C}\right)\right)}$, is generated by the fiber $F$ and by $-K_{\text {rel }}$; hence there exists a very ample curve $C_{\delta}$ in $\mathbb{P}\left(\mathcal{E}_{\mid C}\right)$ satisfying $\tau\left(C_{\delta}\right) \geq \delta$. Since we may assume that $C_{\delta}$ is smooth and, by construction, the normal bundle of $C_{\delta}$ in $\mathbb{P}(\mathcal{E})$ is globally generated, then $C_{\delta}$ is movable in $\mathbb{P}(\mathcal{E})$.

On the other hand, given a movable curve $D$ in $\mathbb{P}(\mathcal{E})$ with $\tau(D)>0$, we will show that $D$ is of the form $\widetilde{C}$, for some $C$. In fact, arguing as in Remark 2.6, we consider the corresponding ruled surface $\mathbb{P}\left(\mathcal{E}_{\mid C}\right)$, where $C$ is the normalization of $\pi(D)$. If $\mathcal{E}_{\mid C}$ is semistable, then $\tau(D) \leq 0$, a contradiction. If $\mathcal{E}_{\mid C}$ is not semistable, then we consider the strict transform $D^{\prime}$ of $D$ in $\mathbb{P}\left(\mathcal{E}_{\mid C}\right)$. If $D^{\prime}$ is movable in $\mathbb{P}\left(\mathcal{E}_{\mid C}\right)$, then $\tau(D)=\tau\left(D^{\prime}\right)<0$; hence we may assume that $D^{\prime}$ is not movable, and therefore $D^{\prime}=\widetilde{C}$. 
We will now make a closer analysis of the positivity of divisors in $\mathbb{P}(\mathcal{E})$ with respect to rational curves in $\mathbb{P}(\mathcal{E})$. We begin with the following straightforward lemma (see, e.g., [Ha2, Chapter V, Section 2]).

\section{LEMMA 3.2}

Let $(X, \mathcal{E})$ be as in Setup 1.1, and let $\mathcal{M}$ be a dominating family of rational curves of $H_{X}$-degree $\mu$ in $X$. Let $D$ be an effective divisor numerically proportional to $-K_{\mathrm{rel}}+b H$, and let $\ell$ be a curve in $\mathcal{M}^{t}, t \geq 0$. Then

- if $b<-t$, then $\mathbb{P}\left(\mathcal{E}_{\mid \ell}\right) \subset D$;

- if $t>b \geq-t$ and $\mathbb{P}\left(\mathcal{E}_{\mid \ell}\right)$ is not contained in $D$, then $\mathbb{P}\left(\mathcal{E}_{\mid \ell}\right) \cap D$ contains the (unique) minimal section $\widetilde{\ell}$, being exactly the section when $b=-t$.

In particular, if $\mathcal{M}^{t}$ dominates $X$ and $b<t$, then every linear system of the form $\left|k\left(-K_{\text {rel }}+b H\right)\right|$ contains a fixed component $F$, where $F$ denotes the closure of $\operatorname{Locus}\left(\widetilde{\mathcal{M}}^{t}\right)$.

A weaker version of this lemma provides a splitting criterion for uniform bundles.

\section{COROLLARY 3.3}

Let $(X, \mathcal{E})$ be as in Setup 1.1. Assume, moreover, that $\mathcal{M}=\mathcal{M}^{t}$ is a uniform unsplit covering family of rational curves on $X$, with $t>0$, and that $H^{0}\left(\mathcal{E}\left(-\left(c_{1}+\right.\right.\right.$ $t) / 2)) \neq 0$. Then $\mathcal{E}$ is decomposable.

Proof

Take $D \in\left|L-\left(\left(c_{1}+t\right) / 2\right) H\right|=\left|1 / 2\left(-K_{\text {rel }}+t H\right)\right|$, and denote by $Z \subset X$ the biggest subset such that $\pi^{-1}(Z) \subset D$, whose cohomology class is $c_{2}\left(\mathcal{E}\left(\left(c_{1}+t\right) / 2\right)\right)$. Hence it suffices to show that $Z$ is empty: assume the contrary. If $\ell \in \mathcal{M}$ is not contained in $Z$, then any nonzero section of $\left.\mathcal{E}\left(\left(c_{1}+t\right) / 2\right)\right)$ does not vanish identically on $\ell$ and hence is nowhere vanishing on $\ell$ since $\mathcal{E}$ has splitting type $\left(\left(c_{1}+t\right) / 2,\left(c_{1}-t\right) / 2\right)$; therefore $\ell \cap Z=\emptyset$. It follows that $\operatorname{ChLocus}_{z}(\mathcal{M}) \subset Z$, for every $z \in Z$, and this contradicts $\operatorname{Pic}(X) \simeq \mathbb{Z}$ and $\mathcal{M}$ unsplit.

\section{REMARK 3.4}

Let us observe that the same result holds in the case $t=0$ without assuming $H^{0}\left(\mathcal{E}\left(-c_{1} / 2\right)\right) \neq 0$ (cf. [AW, Proposition. 1.2]).

In Lemma 3.1 we have seen that $\rho \geq 0$ when $\mathcal{E}$ is semistable. The next proposition shows that the converse is also true and that, moreover, if $\mathcal{E}$ is not semistable then the pseudoeffective cone of $\mathbb{P}(\mathcal{E})$ is completely determined by the maximal destabilizing subsheaf of $\mathcal{E}$.

PROPOSITION 3.5

Let $(X, \mathcal{E})$ be as in Setup 1.1 , let $\beta$ be the minimum integer such that $\mathcal{E}(\beta)$ has nonzero global sections, and let $\left|k\left(-K_{\mathrm{rel}}+b H\right)\right|$ be a nonempty linear system 
in $\mathbb{P}(\mathcal{E})$. Either $b \geq 0$ or $\left|k\left(-K_{\mathrm{rel}}+b H\right)\right|$ has a base component numerically proportional to $-K_{\mathrm{rel}}+\rho H$. In particular, if $\mathcal{E}$ is not semistable, then $\rho=2 \beta+c_{1}<0$.

Proof

Let $\mathcal{M}$ be a dominating family of rational curves in $X$, and denote by $\mu$ its $H_{X^{-}}$ degree. Denote $t:=\tau(\ell), \ell \in \mathcal{M}$ general, and let $F$ be the closure of $\operatorname{Locus}\left(\widetilde{\mathcal{M}}^{t}\right)$.

Assume $b<0$; then $b<0 \leq t$ and Lemma 3.2 above tells us that $\mid k\left(-K_{\text {rel }}+\right.$ $b H) \mid$ has a base component $F$, the closure of $\operatorname{Locus}\left(\widetilde{\mathcal{M}}^{t}\right)$. Moreover, either $F$ or $k\left(-K_{\text {rel }}+b H\right)-F$ are of type $j\left(-K_{\text {rel }}+c H\right)$ with $\rho \leq c<0$.

If $F$ is not of that type, then we may apply the argument above to $\mid k\left(-K_{\text {rel }}+\right.$ $b H)-F \mid$, obtaining that it has a base component $F$. After a finite number of steps, the nonempty linear system $\left|k^{\prime}\left(-K_{\text {rel }}+b^{\prime} H\right)\right|:=\left|k\left(-K_{\text {rel }}+b H\right)-r F\right|$ will not contain $F$ as a base component, contradicting the fact that $b^{\prime}$ will still be smaller than zero.

We may then assume that $F \equiv j\left(-K_{\text {rel }}+c H\right)$ with $\rho \leq c<0$. Arguing in a similar way, we get that every multiple $r F$ of $F$ has $F$ in its base locus; hence it follows that $|r F|$ is zero-dimensional. In particular, $F$ is not big; hence $F$ is numerically proportional to $\left(-K_{\text {rel }}+\rho H\right)$. This proves the first part of the statement.

For the second part, assume that $\mathcal{E}$ is not semistable. Then $\beta<-c_{1} / 2$ by definition, and we may apply the claim above to $\left|1 / 2\left(-K_{\text {rel }}+\left(2 \beta+c_{1}\right) H\right)\right|=$ $|L+\beta H| \neq \emptyset$ to conclude that it has a base component $F$. But $|L+\beta H|$ consists of irreducible unisecant, that is, of relative degree 1 divisors; hence this is possible only when $F \in|L+\beta H|$. It follows that $\rho=2 \beta+c_{1}$.

\section{REMARK 3.6}

The equality $\rho=2 \beta+c_{1}$ holds also for bundles which are semistable but not stable (and so have $\beta=c_{1}=0$ ). In fact $\rho \geq 0$ by Lemma 3.1, but, on the other hand, $\left|-K_{\text {rel }}\right|=|2 L|$ is not empty. This is no longer true for stable bundles: the blowup $Y$ of $\mathbb{P}^{3}$ along a twisted cubic (see $[\mathrm{SW} 2]$ ) is isomorphic to a $\mathbb{P}^{1}$-bundle $\mathbb{P}(\mathcal{E})$ over $\mathbb{P}^{2}$. The exceptional divisor of $Y$ over $\mathbb{P}^{3}$ is not unisecant; hence $\rho \neq 2 \beta+c_{1}$.

The rest of the section is devoted to a Grauert-Mülich-type result for Fano manifolds. The classical Grauert-Mülich theorem (cf. [OSS, II, Section 2]) tells us that the general splitting type of a vector bundle on $\mathbb{P}^{n}$ with respect to a line cannot have gaps of length bigger than one. For other base varieties, one may still control the gaps between the slopes of the Harder-Narasimhan filtration of the restriction of a vector bundle on a manifold to general complete intersection curves (see, for instance [FHS], [MR1], [Fl]). However it was already noted by Hirschowitz in [Hi] that the standard arguments work in a much broader setting.

In the case of dominating families of rational curves on Fano manifolds, a similar result can be obtained as a byproduct of the arguments of this section; we state it here for bundles of rank two. We will make use of the following remark. 


\section{REMARK 3.7}

Let $(X, \mathcal{E})$ be as in Setup 1.1, and let $\mathcal{M}$ be a dominating family of rational curves, satisfying that $\mathcal{M}_{x}$ is irreducible for $x \in X$ general. Let $t:=\tau(\ell), \ell \in \mathcal{M}$ general, and assume $t \neq 0$. Then the closure of $\operatorname{Locus}\left(\widetilde{\mathcal{M}}^{t}\right)$ is $\mathbb{P}(\mathcal{E})$ or a unisecant divisor.

Proof

Assume that $\widetilde{\mathcal{M}}$ does not dominate $\mathbb{P}(\mathcal{E})$, and let $D$ be the closure of $\operatorname{Locus}\left(\widetilde{\mathcal{M}}^{t}\right)$. Let $f$ be a general fiber of $\pi$; by the generality of $f$ we can assume that $\pi(f) \notin$ $\pi\left(D \backslash \operatorname{Locus}\left(\widetilde{\mathcal{M}}^{t}\right)\right)$ and that the intersection of $D$ and $f$ is transversal; by the irreducibility of $\mathcal{M}_{x}$ the intersection number is then one.

The following result may be interpreted as a Grauert-Mülich-type theorem for rational curves on Fano manifolds.

PROPOSITION 3.8

Let $(X, \mathcal{E})$ be as in Setup 1.1. Let $\mathcal{M}$ be a dominating family of rational curves on $X$ such that $\mathcal{M}_{x}$ is irreducible for the general $x \in X$. Let $\mu$ denote the $H_{X-}$ degree of $\mathcal{M}$. Either $\tau(\ell) \leq 1 / \mu$ for the general $\ell \in \mathcal{M}$, or $\mathcal{E}$ is destabilized by a line bundle of degree $\beta=-\left(\tau(\ell)+c_{1}\right) / 2$.

Proof

Set $t:=\tau(\ell), \ell \in \mathcal{M}$ general, assume $t \geq 2 / \mu$, and consider the corresponding families $\mathcal{M}^{t}$ and $\widetilde{\mathcal{M}}^{t}$. We will prove that the evaluation morphism $\widetilde{\mathrm{ev}}^{t}: \widetilde{\mathcal{U}}^{t} \cong$ $\mathcal{U}^{t} \rightarrow \mathbb{P}(\mathcal{E})$ is not dominant. Then, by Remark 3.7, the closure of Locus $\left(\widetilde{\mathcal{M}}^{t}\right)$ will be a unisecant divisor, corresponding to an element in $\operatorname{Hom}_{X}\left(\mathcal{O}_{X}(-b), \mathcal{E}\right)$ for some $b$. Restricting to $\ell$ we see that $-b=\left(\tau(\ell)+c_{1}\right) / 2$. Moreover, since $\mathcal{M}^{t}$ covers $X, H^{0}\left(X, \mathcal{E}\left(b^{\prime}\right)\right)=0$ for $b^{\prime}<b$. This concludes the proof.

In order to see that $\widetilde{\mathrm{ev}}^{t}$ is not dominant, note that for the general element $\ell$ of $\mathcal{M}^{t}$, the restriction of the tangent bundle of $X$ to $\ell$ is nef. Since $d\left(\mathrm{ev}^{t}\right)_{\mid \ell}$ : $\left(T_{\mathcal{U}^{t}}\right)_{\ell} \rightarrow\left(T_{X}\right)_{\mid \ell}$ is the evaluation of global sections of $\left(T_{X}\right)_{\mid \ell}$, it follows that its kernel satisfies $\left(T_{\mathcal{U}^{t} \mid X}\right)_{\mid \ell} \cong \mathcal{O}(-1) \oplus \cdots \oplus \mathcal{O}(-1)$. On the other hand $\left(T_{\mathbb{P}(\mathcal{E}) \mid X}\right)_{\mid \ell} \cong$ $\mathcal{O}(t \mu)$, with $t \mu \geq 2$ by assumption; hence there are no nonzero morphisms from $\left(T_{\mathcal{U}^{t} \mid X}\right)_{\mid \ell}$ to $\left(T_{\mathbb{P}(\mathcal{E}) \mid X}\right)_{\mid \ell}$, and $d\left(\widetilde{\mathrm{ev}}^{t}\right)_{\mid \ell}$ is not generically surjective.

\section{The pseudoeffective cone of $\mathbb{P}(\mathcal{E})$}

In this section we will explore the relation between $\tau(\mathcal{E})$ and $\rho(\mathcal{E})$. Unless otherwise stated we will always assume the following.

\section{SETUP 4.1}

$(X, \mathcal{E})$ will be as in Setup 1.1 with $b_{4}(X)=1$. As described in Section 1.1 we will use the following equalities of numerical classes of cycles: $c_{2}(\mathcal{E})=c_{2} \Sigma$ and $H_{X}^{2}=d \Sigma$ with $c_{2}, d \in \mathbb{Z}, d>0 ; \Delta(\mathcal{E})=\left(d c_{1}^{2}-4 c_{2}\right) \Sigma=: d \Delta \Sigma$ and $K_{\text {rel }}^{2}=\Delta H^{2}$. 
The main idea we will use is the following.

\section{REMARK 4.2}

Pseudoeffective divisors have nonnegative intersection with movable classes of 1-cycles, for instance, with complete intersections of nef divisors. Thus we have the following restrictions:

(2) $\left(-K_{\text {rel }}+\rho^{\prime} H\right) \cdot\left(-K_{\text {rel }}+\tau H\right)^{j} \cdot H^{n-j} \geq 0, \quad$ for all $j \in\{0, \ldots, n\}, \rho^{\prime} \geq \rho$.

By the Chern-Wu relation $K_{\text {rel }}^{2}=\Delta H^{2}$, the equality $H^{n+1}=0$, and the inequality $-K_{\text {rel }} H^{n}>0$, inequality (2) reduces, for each $j$, to

$$
\frac{\left(\rho^{\prime}+\sqrt{\bar{\Delta}}\right)(\tau+\sqrt{\bar{\Delta}})^{j}-\left(\rho^{\prime}-\sqrt{\Delta}\right)(\tau-\sqrt{\Delta})^{j}}{\sqrt{\Delta}} \geq 0, \quad j \in\{0, \ldots, n\}, \rho^{\prime} \geq \rho,
$$

if $\Delta \neq 0$, and to

$$
\tau^{j-1}\left(\tau+j \rho^{\prime}\right) \geq 0, \quad \text { for all } j \in\{0, \ldots, n\}, \rho^{\prime} \geq \rho,
$$

if $\Delta=0$. In this case, applying Theorem 2.3, Lemma 2.5, and Proposition 3.5, all the inequalities reduce to

$$
\tau+n \rho \geq 0
$$

\subsection{The case $\Delta<0$}

Denoting by $\arg (z) \in[0,2 \pi)$ the argument of a complex number $z \neq 0,(3)$ is, in this case, equivalent to

$$
\arg \left(\left(\rho^{\prime}+i \sqrt{-\Delta}\right)(\tau+i \sqrt{-\Delta})^{j}\right) \leq \pi, \quad \text { for all } j \in\{0, \ldots, n\} .
$$

Note that the inequality for $j=n$ implies the rest; therefore (3) is equivalent to

$$
\arg \left(\left(\rho^{\prime}+i \sqrt{-\Delta}\right)(\tau+i \sqrt{-\Delta})^{n}\right) \leq \pi .
$$

Applying (6) above to $\rho^{\prime}=\tau$, we get the following inequality, which can be read as the positivity of certain Schur polynomials of the minimal nef twist of $\mathcal{E}$, and which can be interpreted as a lower bound for $\tau$ in terms of the discriminant $\Delta$ and the invariants of $X$.

\section{LEMMA 4.3}

Let $(X, \mathcal{E})$ be as in Setup 4.1. Then

$$
c_{1}^{2}<\frac{4 c_{2}}{d} \leq c_{1}^{2}+\tau^{2} \tan ^{2}\left(\frac{\pi}{n+1}\right) .
$$

We obtain stronger restrictions by applying equation (6) to $\rho^{\prime}=\rho$. However, it is not clear whether $\rho<\tau$. We already know that this occurs when $\mathcal{E}$ is not semistable (see Theorem 2.3, Proposition 3.5). The next proposition shows that this is also the case if $\tau$ is rational and $n \neq 2,3,5$. 


\section{PROPOSITION 4.4}

Let $(X, \mathcal{E})$ be as in Setup 4.1, $\mathcal{E}$ nontrivial. If $\tau \in \mathbb{Q}$, then $-K_{\text {rel }}+\tau H$ is big unless $\Delta<0$ and $n=2,3$, and 5. In particular, $\rho<\tau$.

Proof

Assume that $-K_{\text {rel }}+\tau H$ is not big. On one hand, we get $\rho=\tau$; hence $\rho \geq 0$ by Theorem 2.3 , so $\mathcal{E}$ is semistable by Proposition 3.5 and $\Delta \leq 0$. On the other hand, we obtain an equality $\left(-K_{\text {rel }}+\tau H\right)^{n+1}=0$ that, arguing as above, leads us to $\tau=0$, and to the triviality of $\mathcal{E}$ by Theorem 2.3 , if $\Delta=0$.

If $\Delta<0$, then we get

$$
\tan ^{2}\left(\frac{\pi}{n+1}\right)=\frac{-\Delta}{\tau^{2}} \in \mathbb{Q}
$$

The algebraic degree of $\tan (\pi /(n+1))$ over $\mathbb{Q}$ is classically known (see $[\mathrm{Ni}$, pp. 33-41]; see also [Ca, Proposition 2]), and one may check directly that the only possible values of $n$ are 2,3 , or 5 .

\subsection{The case $\Delta \geq 0$}

In this case $\mathcal{E}$ is not semistable, unless $\mathcal{E} \simeq \mathcal{O}_{X} \oplus \mathcal{O}_{X}$ (cf. Lemma 2.5); hence, by Proposition 3.5 and Remark 3.6, we know that $\rho=2 \beta+c_{1} \leq 0$ and $c_{2}(\mathcal{E}(\beta)) \geq 0$. In particular, since the numerical classes $c_{2}(\mathcal{E}(k))=\left(c_{2}+d k c_{1}+d k^{2}\right) \Sigma$ and $c_{2}\left(\mathcal{E}\left(-c_{1}-k\right)\right)$ are equal, we get

$$
\rho=2 \beta+c_{1} \leq-\sqrt{\Delta} .
$$

\section{REMARK 4.5}

Equality in (7) holds if and only if $\mathcal{E}$ is a direct sum of line bundles. In fact if equality holds, then $c_{2}(\mathcal{E}(\beta))=0$ and $\mathcal{E}$ splits. The converse follows from a direct computation.

On the other hand, the set of restrictions (3) provide the following.

\section{LEMMA 4.6}

Let $(X, \mathcal{E})$ be as in Setup 4.1 with $\Delta \geq 0$. Then

$$
\begin{aligned}
\tau & \geq \sqrt{\Delta}, \quad \text { and } \\
-\sqrt{\Delta}-\varepsilon & \leq \rho \leq-\sqrt{\Delta},
\end{aligned}
$$

where

$$
\varepsilon=\frac{2 \sqrt{\Delta}(\tau-\sqrt{\Delta})^{n}}{(\tau+\sqrt{\Delta})^{n}-(\tau-\sqrt{\Delta})^{n}} \quad \text { if } \Delta>0 \text { and } \varepsilon=\frac{\tau}{n} \text { if } \Delta=0 \text {. }
$$

Proof

With the same notation as in Remark 4.2, if $\Delta>0$, inequalities (3) give

$$
(\rho+\sqrt{\Delta})(\tau+\sqrt{\Delta})^{j} \geq(\rho-\sqrt{\Delta})(\tau-\sqrt{\Delta})^{j} \text { for all } j \leq n .
$$


By (7), $\rho+\sqrt{\Delta} \leq 0$; therefore $\rho-\sqrt{\Delta}<\rho+\sqrt{\Delta} \leq 0$, and the previous inequality tells us in particular that $\tau \geq \sqrt{\Delta}$ : otherwise

$$
(\rho+\sqrt{\Delta})(\tau+\sqrt{\Delta}) \leq(\rho+\sqrt{\Delta})(\tau-\sqrt{\Delta})<(\rho-\sqrt{\Delta})(\tau-\sqrt{\Delta}) .
$$

Finally, since $\tau-\sqrt{\Delta} \geq 0 \geq \rho+\sqrt{\Delta}$, the set of inequalities in (3) reduces to the one obtained for $j=n$, and a simple computation concludes the proof.

If $\Delta=0$, then (8) follows from Theorem 2.3 and (9) is a restatement of (4).

\section{REMARK 4.7}

Equality in (8) holds if and only if $\mathcal{E}$ is a direct sum of line bundles. In fact, if $\tau=\sqrt{\Delta}>0$, then $\varepsilon=0$, equality holds in (7), and $\mathcal{E}$ splits by Remark 4.5. If $\tau=\Delta=0$, the same result follows from Theorem 2.3. The converse follows from a direct computation.

Bounding $\varepsilon \leq \varepsilon^{\prime}:=(\tau-\sqrt{\Delta}) / n$ we obtain the following inequality.

COROLLARY 4.8

Let $(X, \mathcal{E})$ be as in Setup 4.1 with $\Delta \geq 0$. Then

$$
\tau+n \rho+(n-1) \sqrt{\Delta} \geq 0 .
$$

Finally, we obtain the following two splitting criteria.

\section{COROLLARY 4.9}

Let $(X, \mathcal{E})$ be as in Setup 4.1 with $\Delta \geq 0$, and consider the following interval:

$$
I=\left[-\frac{c_{1}+\sqrt{\Delta}}{2}-\frac{\varepsilon^{\prime}}{2},-\frac{c_{1}+\sqrt{\Delta}}{2}\right] .
$$

Then

- $I \cap \mathbb{Z} \neq \emptyset$, and

- if $I \cap \mathbb{Z}=-\left(c_{1}+\sqrt{\Delta}\right) / 2$, then $\mathcal{E}$ splits as a direct sum of line bundles.

\section{COROLLARY 4.10}

Let $(X, \mathcal{E})$ be as in Setup 4.1 with $\Delta \geq 0$. If $-\left(c_{1}+\sqrt{\Delta}\right) / 2 \in \mathbb{Z}$ and

$$
\tau<2 n+\sqrt{\Delta}
$$

then $\mathcal{E}$ is decomposable.

Proof

It is immediate to see that $\sqrt{\Delta}$ is an integer if and only if $-\left(c_{1}+\sqrt{\Delta}\right) / 2 \in \mathbb{Z}$; then by Corollary 4.9 it suffices to check that $\varepsilon^{\prime}<2$.

We finish this section by discussing the effects on $\overline{\operatorname{Eff}(\mathbb{P}(\mathcal{E}))}$ of assuming that $-K_{\text {rel }}+\tau H$ is semiample. More concretely, we will prove that if the associate 
contraction is of fiber type or divisorial, then $\rho$ is completely determined from the rest of the invariants of $(X, \mathcal{E})$. We will make use of the following observation on the set of inequalities (2), whose proof follows from a straightforward analysis of the different forms that (2) takes according to the sign of $\Delta$.

LEMMA 4.11

Let $(X, \mathcal{E})$ be as in Setup 4.1, and assume that $\mathcal{E}$ is indecomposable. Then

$$
\left(-K_{\mathrm{rel}}+\rho^{\prime} H\right) \cdot\left(-K_{\mathrm{rel}}+\tau H\right)^{j} \cdot H^{n-j}>0,
$$

for every $\rho^{\prime} \geq \rho$ unless $\left(j, \rho^{\prime}\right)=(n, \rho)$.

PROPOSITION 4.12

Let $(X, \mathcal{E})$ be as in Setup 4.1, with $\mathcal{E}$ indecomposable. Assume further that there exists a morphism $\varphi: \mathbb{P}(\mathcal{E}) \rightarrow Y$ contracting the second extremal ray $R_{2}$ of $\mathbb{P}(\mathcal{E})$. Then

- if $\varphi$ is of fiber type, then $\mathcal{E}$ is stable, $\rho=\tau$, and $n=\operatorname{dim} Y=2,3$, or 5 .

- if $\varphi$ is divisorial, then $\operatorname{dim} \varphi(\operatorname{Exc}(\varphi))=n-1$ and $\rho$ is determined by $\tau$ and $\Delta$. In particular, if $\Delta<0$, then

$$
\arg \left((\rho+i \sqrt{-\Delta})(\tau+i \sqrt{-\Delta})^{n}\right)=\pi
$$

Proof

If $\varphi$ is a fiber-type contraction, then $-K_{\text {rel }}+\tau H$ is not big; hence $\rho=\tau$ and, by Theorem 2.3, Proposition 3.5, and Remark 3.6, $\mathcal{E}$ is stable. In particular, $\Delta<0$ and we can apply Proposition 4.4 to get $n=2,3$, or 5 . Finally, Lemma 4.11 provides $\left(-K_{\text {rel }}+\tau H\right)^{n} \cdot H>0$, which forces $\operatorname{dim} Y=n$.

If $\varphi$ is a divisorial contraction, then the class of $\operatorname{Exc}(\varphi)$ is effective and not big; hence it is numerically proportional to $-K_{\mathrm{rel}}+\rho H$. Therefore we have

$$
\begin{gathered}
\left(-K_{\mathrm{rel}}+\rho H\right) \cdot\left(-K_{\mathrm{rel}}+\tau H\right)^{n-1} \cdot H>0 \quad \text { and } \\
\left(-K_{\mathrm{rel}}+\rho H\right) \cdot\left(-K_{\mathrm{rel}}+\tau H\right)^{n}=0 .
\end{gathered}
$$

The first expression tells us that $\operatorname{dim} \varphi(\operatorname{Exc}(\varphi)) \geq n-1$, whereas, arguing as above, the reduction modulo $K_{\text {rel }}^{2}=\Delta H^{2}$ of the second shows that $\rho$ is uniquely determined by the values of $\tau$ and $\Delta$. In particular, when $\Delta<0$, the relation between $\rho, \Delta$, and $\tau$ is equation (11).

\section{Splitting criteria}

Throughout this section we will use the following notation and assumptions unless otherwise stated.

\section{SETUP 5.1}

Let $(X, \mathcal{E})$ be as in Setup 4.1. We denote by $\mathcal{M}$ a dominating family of rational curves on $X$ of $H_{X}$-degree $\mu$. 


\section{REMARK 5.2}

By definition, the zero locus of a section of $\mathcal{E}(\beta)$ has pure codimension 2 ; hence a general element $\ell_{0}$ of $\mathcal{M}$ avoids it (cf. [K, II, Proposition 3.7]), so that $\mathcal{E}_{\mid \ell_{0}}$ is an extension of $\mathcal{O}\left(\left(c_{1}+\beta\right) \mu\right)$ by $\mathcal{O}(-\beta \mu)$. In particular, if $\mathcal{E}$ is not stable, then for all $\ell \in \mathcal{M}$ we have

$$
\tau(\ell) \geq \tau\left(\ell_{0}\right)=-\left(2 \beta+c_{1}\right)=-\rho,
$$

where the last equality follows from Proposition 3.5 and Remark 3.6.

The following two results are based on [APW, Theorem 10.5] and [B, Theorem 1].

\section{LEMMA 5.3}

Let $(X, \mathcal{E})$ be as in Setup 5.1; assume that, for some rational number $t$, there is a surface $S \subset \mathbb{P}(\mathcal{E})$ such that $\pi_{\mid S}$ is finite and that $\left(-K_{\mathrm{rel}}+t H\right) \cdot C=0$ for every curve $C \subset S$. Then $\Delta=t^{2}$.

\section{Proof}

By hypothesis, $\left(K_{\mathrm{rel}}\right)_{\mid S}$ and $t H_{\mid S}$ are numerically equivalent. Hence $\left(\left(K_{\mathrm{rel}}\right)_{\mid S}\right)^{2}=$ $t^{2} H_{\mid S}^{2}$. But $\left(K_{\text {rel }}\right)^{2}$ is numerically equivalent to $\Delta H^{2}$ and $\pi_{\mid S}$ is finite, therefore $\Delta=t^{2}$.

The numerical conditions of the previous lemma lead to a splitting criterion.

\section{LEMMA 5.4}

Let $(X, \mathcal{E})$ and $\mathcal{M}$ be as in Setup 5.1. If $\Delta=t^{2}$ for some positive rational number $t$ and there exists a curve $\ell \in \mathcal{M}$ satisfying $\tau(\ell) \leq t$, then $\mathcal{E}$ is decomposable.

Proof

Since $\Delta \geq 0$, then, by Bogomolov inequality and Lemma $2.5, \mathcal{E}$ is not semistable; the assumption on $\tau(\ell)$ yields, by (12), that $\rho \geq-\sqrt{\Delta}$; combining it with (7) we get $\rho=-\sqrt{\Delta}$, and $\mathcal{E}$ is decomposable by Remark 4.5.

A more general splitting criterion can be stated as follows.

\section{COROLLARY 5.5}

Let $(X, \mathcal{E})$ and $\mathcal{M}$ be as in Setup 5.1. Assume that there exists a rational number $t$ such that

$$
\widetilde{\mathcal{M}}_{y}^{t} \text { contains a complete curve } T \text { for some } y \in \mathbb{P}(\mathcal{E}) \text {. }
$$

Then $\mathcal{E}$ is decomposable.

Proof

Let $S$ be the locus of curves parameterized by the complete curve $T$. Every curve in $S$ is numerically proportional to a curve $\ell$ of $\widetilde{\mathcal{M}}_{y}^{t}$. Since $\tau(\ell)=t$ it follows that 
$-K_{\text {rel }}+t H$ is numerically trivial on $H$; hence $\Delta=t^{2}$ by Lemma 5.3 , and the splitting follows from Lemma 5.4.

\section{REMARK 5.6}

The same arguments work if we assume that for some $t$, the family $\widetilde{\mathcal{M}}^{t}$ is unsplit and for some $y \in \operatorname{Locus}\left(\widetilde{\mathcal{M}}^{t}\right)$ there is a component of $\operatorname{Chlocus}_{y}\left(\widetilde{\mathcal{M}}^{t}\right)$ of dimension $\geq 2$.

Keeping track of the dimensions of the families parameterizing rational curves of fixed splitting type passing by a general point, one may translate condition (13) into a numerical splitting criterion.

\section{THEOREM 5.7}

Let $(X, \mathcal{E})$ and $\mathcal{M}$ be as in Setup 5.1, and assume that $\mathcal{M}_{x}$ is proper for a general $x \in X$ and that $\mathcal{E}$ is not stable. Assume, moreover, that

- $\tau<2 i_{X}-\rho-4 / \mu$ if $\mathcal{E}$ is not semistable, and

- $\tau<2 i_{X}-6 / \mu$ if $\mathcal{E}$ is semistable.

Then $\mathcal{E}$ is decomposable.

Proof

Let $x$ be a general point in $X$, and denote by $t_{\min }$ (resp., $t^{\max }$ ) the minimum (resp., the maximum) integer such that $\mathcal{M}_{x}^{t_{\min }} \neq \emptyset$ (resp., $\mathcal{M}_{x}^{t^{\max }} \neq \emptyset$ ). By Remark 5.2, $t_{\min }=-\rho \geq 0$. Since $\mathcal{E}$ is not stable, by Proposition 3.5 and Remark 3.6 we have $\rho=2 \beta+c_{1}$. Let $D$ be a divisor in $|L+\beta H|=\mid \frac{1}{2}\left(-K_{\text {rel }}+\right.$ $\rho H) \mid$, and let $t \in\left[t_{\text {min }}, t^{\max }\right]$. By Lemma 3.2 we deduce that $\operatorname{Locus}\left(\widetilde{\mathcal{M}}^{t}\right) \subseteq D$ unless $t=t_{\text {min }}=\rho=0$.

Assume first that $\mathcal{E}$ is not semistable. The divisor $D$ meets the fiber over a general $x \in X$ in a point; hence, from the argument above, we get that the existence of a complete curve in $\mathcal{M}_{x}^{t}$ for some $t$, implies condition (13). Since $\mathcal{M}_{x}$ is proper, we may thus express a necessary condition for the bundle not to split as follows (cf. [APW, Corollary 10.6]):

(a) $\operatorname{dim} \mathcal{M}_{x}^{t^{\max }}=0$,

(b) $\operatorname{dim} \overline{\mathcal{M}_{x}^{t}}-\operatorname{dim}\left(\overline{\mathcal{M}_{x}^{t}} \cap\left(\bigcup_{b>t} \mathcal{M}_{x}^{b}\right)\right) \leq 1$ if $t_{\min } \leq t<t^{\max }$.

In particular, $\#\left\{\mathcal{M}_{x}^{t}\right\} \geq \operatorname{dim} \mathcal{M}_{x}+1=i_{X} \mu-1$. On the other hand,

$$
\#\left\{\mathcal{M}_{x}^{t}\right\} \leq \frac{\mu}{2}\left(t^{\max }-t_{\text {min }}\right)+1 \leq \frac{\mu}{2}(\tau+\rho)+1
$$

which combined with the previous equation, gives the first part of the statement.

The case of $\mathcal{E}$ semistable but not stable (which corresponds to $t_{\min }=\rho=0$ ) is slightly different: $\operatorname{since} \operatorname{Locus}\left(\widetilde{\mathcal{M}}^{0}\right) \not \subset D$, we only know that if $\mathcal{E}$ is indecomposable, then $\#\left\{\mathcal{M}_{x}^{t}\right\} \geq \operatorname{dim} \mathcal{M}_{x}=i_{X} \mu-2$, and we conclude by combining this with (14). 


\section{COROLLARY 5.8}

With the same notation as above, assume that $\Delta \geq 0$ and that $\tau<2 i_{X}+\sqrt{\Delta}-$ $4 / \mu$. Then $\mathcal{E}$ is decomposable.

\section{Proof}

By Bogomolov inequality and Lemma 2.5, we may assume that $\mathcal{E}$ is not semistable. By (7), we have $\sqrt{\Delta} \leq-\rho$ and we conclude by Theorem 5.7 .

The last statement of this section shows that decomposability is determined by the pair $(\tau, \rho)$.

\section{COROLLARY 5.9}

Let $(X, \mathcal{E})$ be as in Setup 4.1; then $\mathcal{E}$ is decomposable if and only if $\tau+\rho=0$.

Proof

Assume that $\tau+\rho=0$; by Theorem 2.3 we may assume that $\tau>0$. Hence, by Lemma $3.1, \mathcal{E}$ is not semistable. Consider a minimal covering family of rational curves $\mathcal{M}$, of $H_{X}$-degree $\mu$. By Theorem 5.7 the bundle is decomposable unless possibly if $i_{X}=2$ and $\mu=1$ (hence $\mathcal{M}$ is unsplit). In this case, by Remark 5.2, $\mathcal{E}$ is uniform, and the decomposability follows from Corollary 3.3. The converse is a direct computation.

\section{Fano bundles}

In this section we will apply our techniques to Fano bundles, that is, bundles whose projectivization is a Fano manifold, in order to obtain structure theorems and partial classification results. Notice that a bundle $\mathcal{E}$ on $X$ is a Fano bundle if and only if $\tau<i_{X}$.

We begin by describing the second contraction $\varphi: \mathbb{P}(\mathcal{E}) \rightarrow Y$. We will say that $\varphi$ is a $\mathbb{P}^{1}$-bundle if there is a rank two vector bundle $\mathcal{F}$ on $Y$ such that $\mathbb{P}(\mathcal{E})=\mathbb{P}(\mathcal{F})$; we will say that $\varphi$ is a conic bundle if there is a rank three vector bundle $\mathcal{F}$ on $Y$ such that $\mathbb{P}(\mathcal{E})$ embeds in $\mathbb{P}(\mathcal{F})$ as a divisor of relative degree two. We will denote by $l\left(R_{2}\right)$ the length of the extremal ray $R_{2}$, that is, the minimum anticanonical degree of rational curves whose numerical class is in $R_{2}$, and by $C$ a curve in the ray whose anticanonical degree equals the length. The length of a $\mathbb{P}^{1}$-bundle contraction is two, while the length of a conic bundle contraction is either one if there are singular fibers or two if all the fibers are reduced and irreducible.

\section{LEMMA 6.1}

Let $(X, \mathcal{E})$ be as in Setup 4.1, and assume that $\mathcal{E}$ is Fano. If $\mathcal{E}$ is indecomposable then, with the same notation as above:

(1) either $\varphi$ is a $\mathbb{P}^{1}$-bundle, $l\left(R_{2}\right)=2, \tau=i_{X}-\frac{2}{H \cdot C}$, or

(2) $\varphi$ is a conic bundle with reducible fibers, $l\left(R_{2}\right)=1, \tau=i_{X}-\frac{1}{H \cdot C}$, or 
(3) $\varphi$ is the blowup of a codimension two smooth subvariety, $l\left(R_{2}\right)=1, \tau=$ $i_{X}-\frac{1}{H \cdot C}$.

In all cases $Y$ is smooth and Fano.

Proof

Observe first that any nontrivial fiber of $\varphi$ is one-dimensional. In fact, if a fiber contained a surface, we would have $\Delta=\tau^{2}$ by Lemma 5.3 , and $\mathcal{E}$ would split by Remark 4.7. By [W, Theorem 1.2] we then get that $Y$ is smooth, and either $l\left(R_{2}\right)=1$ and we are in case $(2),(3)$ or $l\left(R_{2}\right)=2$ and $\varphi: \mathbb{P}(\mathcal{E}) \rightarrow Y$ is a conic bundle without reducible fibers. In this case we conclude by Lemma 6.2. Moreover, $Y$ is always Fano, since it is covered by rational curves and its Picard number is one.

\section{LEMMA 6.2}

With the same notation as above, assume that $\varphi: \mathbb{P}(\mathcal{E}) \rightarrow Y$ is a smooth conic bundle. Then $\varphi$ is a $\mathbb{P}^{1}$-bundle.

\section{Proof}

Denote by $-K^{\prime}$ the relative anticanonical divisor of the morphism $\varphi: \mathbb{P}(\mathcal{E}) \rightarrow Y$; by $H^{\prime}$ the pullback via $\varphi$ of $H_{Y}$, the ample generator of $\operatorname{Pic}(Y)$; and by $i_{Y}$ the index of $Y$. Let $f$ and $f^{\prime}$ be the numerical class of the fibers of $\pi$ and $\varphi$, respectively, and set $\mu:=H \cdot f^{\prime}, \mu^{\prime}:=H^{\prime} \cdot f$. For simplicity, we will denote $\nu:=$ $i_{X} \mu-2=K \cdot f^{\prime}, \nu^{\prime}:=i_{Y} \mu^{\prime}-2=K^{\prime} \cdot f$. We then have the following intersection numbers:

\begin{tabular}{|c|c|c|c|c|}
\hline & $-K$ & $H$ & $-K^{\prime}$ & $H^{\prime}$ \\
\hline$f$ & 2 & 0 & $-\nu^{\prime}$ & $\mu^{\prime}$ \\
\hline$f^{\prime}$ & $-\nu$ & $\mu$ & 2 & 0 \\
\hline
\end{tabular}

which allow us to write

$$
\left(\begin{array}{c}
-K \\
H
\end{array}\right)=\left(\begin{array}{cc}
-\frac{\nu}{2} & \frac{4-\nu \nu^{\prime}}{2 \mu^{\prime}} \\
\frac{\mu}{2} & \frac{\nu^{\prime} \mu}{2 \mu^{\prime}}
\end{array}\right)\left(\begin{array}{c}
-K^{\prime} \\
H^{\prime}
\end{array}\right)
$$

Assume that $\varphi$ is not a $\mathbb{P}^{1}$-bundle. Equivalently, $\left\{-K^{\prime}, H^{\prime}\right\}$ is a base of $\operatorname{Pic}(\mathbb{P}(\mathcal{E}))$; therefore the determinant of the base-change matrix is \pm 2 , and we get $\mu=2 \mu^{\prime}$. Using this base change, together with the equality

$$
-\Delta=\tau^{2} \tan ^{2}\left(\frac{\pi}{n+1}\right)=\left(\frac{\nu}{\mu}\right)^{2} \tan \left(\frac{\pi}{n+1}\right)
$$

obtained as in Proposition 4.4, the Chern-Wu relation $K^{2}=\Delta H^{2}$ transforms into

$$
K^{\prime 2}=2 \frac{\nu \nu^{\prime}(1+a)-4}{\nu \mu^{\prime}(1+a)} K^{\prime} H^{\prime}-\frac{16-8 \nu \nu^{\prime}+\nu^{2} \nu^{\prime 2}(1+a)}{\nu^{2} \mu^{\prime 2}(1+a)} H^{\prime 2}
$$

where $a$ denotes $\tan ^{2}(\pi /(n+1))$. 
It is known (see, for instance, [S]) that being $\varphi$ a conic bundle, the cohomology class of the closed set of points in $Y$ whose inverse image by $\varphi$ is a singular conic is $-\varphi_{*}^{\prime}\left(K^{\prime 2}\right)$. By hypothesis, $\varphi$ is smooth; hence the intersection number $K^{\prime 2} H^{\prime n-1}$ is equal to zero. Using (16) and the obvious equalities $H^{\prime n+1}=0$, $-K^{\prime} H^{\prime n}=2 H_{Y}^{n}$, we then have

$$
\nu \nu^{\prime}=\frac{4}{1+a}=4 \cos \left(\frac{\pi}{n+1}\right)=1,2,3 \text { for } n=2,3,5, \text { respectively. }
$$

Since $\nu=i_{X} \mu-2=2\left(i_{X} \mu^{\prime}-1\right)$ is even, we conclude that the only possibility is $n=3, i_{X}=2, i_{Y}=3$, and $\mu^{\prime}=1$. In this case, we have $-K=K^{\prime}+H^{\prime}, H=$ $-K^{\prime}+H^{\prime}$, and the Chern-Wu relation reads as $K^{\prime 2}=-H^{\prime 2}$, from which we obtain $-K H^{3}=-4 K^{\prime} H^{\prime 3}$. In particular, $X$ has degree 8 , contradicting the fact that it is a del Pezzo 3 -fold of index two (cf. [F]).

We will now show that, with one exception, indecomposable Fano bundles are stable. Note that it was conjectured by Grauert and Schneider (actually they provided an incomplete proof; cf. [GS]) that every indecomposable rank two vector bundle on $\mathbb{P}^{n}, n \geq 4$, is semistable.

\section{THEOREM 6.3}

Let $(X, \mathcal{E})$ be as in Setup 4.1, and assume that $\mathcal{E}$ is Fano, indecomposable, and not stable; then $X \simeq \mathbb{P}^{2}$ and $\mathcal{E}$ is a bundle with $c_{1}=0, c_{2}=1$ whose projectivization is the blowup of a smooth three-dimensional quadric along a line.

\section{Proof}

Let $\mathcal{M}$ be a minimal covering family of rational curves on $X$ of $H_{X}$-degree equal to $\mu$. Assume first that $\mathcal{E}$ is not semistable; by Proposition 3.5 we have $\rho<0$; hence, by Theorem $5.7, \mathcal{E}$ is decomposable except possibly when $\rho=-1$, $\mu=1$, and $i_{X}=2$. In this case, by Remark $5.2,2=i_{X}>\tau \geq \tau(\ell) \geq 1$ for every $\ell \in \mathcal{M}$; hence $\mathcal{E}$ is uniform of splitting type $(-1,0)$, and we may conclude by Corollary 3.3.

Assume now that $\mathcal{E}$ is semistable. By Remark 3.6 we have $\rho=0$. Let $C$ be a minimal rational curve generating the second extremal ray of $\mathbb{P}(\mathcal{E})$. A divisor $E \in|L|$ is not nef; otherwise we have $\tau=0$ and $\mathcal{E}$ splits by Theorem 2.3. In particular, $E$ has negative intersection with $C$; hence the second contraction $\varphi: \mathbb{P}(\mathcal{E}) \rightarrow Y$ is a smooth blowup with exceptional locus $E$, by Lemma 6.1 ; in particular, $E \cdot C=-1$.

On the other hand, $\beta=0$ implies that $c_{2}>0$, so that $\Delta<0$ and we may apply Proposition 4.12 to get

$$
\frac{4 c_{2}}{d}=-\Delta=\tau^{2} \tan ^{2}\left(\frac{\pi}{2 n}\right) .
$$

As in the proof of Proposition 4.4, we now use [Ni] and [Ca, Proposition 2] to get that $n$ is equal to 2 or 3 . Moreover, from $l\left(R_{2}\right)=1$ and $E \cdot C=-1$ we get that either $\mu=1$ (hence $\tau=i_{X}-1$ ) and $i_{X}=3$ or $\mu=3$ and $i_{X}=1$. 
If $n=3$, since a Fano 3 -fold with Picard number one is covered either by lines or by conics, we may assume $i_{X}=3$. Then $X$ is a smooth quadric; hence $d=2$, and, from (17) we get $2 c_{2}=\frac{4}{3}$, which is impossible.

If $n=2$, then $X$ is $\mathbb{P}^{2}$. From $\tau=2$ we get that $L+H$ is nef and trivial on the second ray; hence $L+H=\varphi^{*} H_{Y}$ for a line bundle $H_{Y}$ on $Y$; since $H_{Y} \cdot f=1$ this line bundle is the generator of $\operatorname{Pic}(Y)$. Computing the canonical bundle of $\mathbb{P}(\mathcal{E})$ with the blowup formula we get that $i_{Y}=3$, that is, $Y \simeq \mathbb{Q}^{3}$. The exceptional divisor $E$ contains a fiber of $\pi$, since $c_{2}=1$ and $\mathcal{E}$ is semistable. The center of the blowup contains the image in $Y$ of this fiber, which is a line $\ell$ in $\mathbb{Q}^{3}$; since the center is smooth, then the center is $\ell$.

The main result of this section is a characterization of bundles $\mathcal{E}$ whose projectivization $\mathbb{P}(\mathcal{E})$ has a second contraction which is a $\mathbb{P}^{1}$-bundle. We will first recall the following.

\section{EXAMPLE 6.4}

Let $K\left(G_{2}\right)$ be a 5 -dimensional Fano homogeneous contact manifold of type $G_{2}$. $K\left(G_{2}\right)$ is a Fano manifold with Picard number one, index three and $b_{4}=1$ which is a linear section of the Grassmannian $\mathbb{G}(1,6)$ with a $\mathbb{P}^{13}$ (see [M, Example 1]). Since $K\left(G_{2}\right)$ is covered by lines, the restriction of the universal quotient bundle $\mathcal{Q}$ on $\mathbb{G}(1,6)$ is a Fano bundle on $K\left(G_{2}\right)$, with $\tau=1$. The projectivization of $\left.\mathcal{Q}\right|_{K\left(G_{2}\right)}$ has a second $\mathbb{P}^{1}$-bundle structure, over a five-dimensional smooth quadric $\mathbb{Q}^{5}$, which corresponds to the projectivization of a Cayley bundle $\mathcal{C}$ (see $[\mathrm{O}, 1.3]$ ); via this description $\mathbb{P}\left(\left.\mathcal{Q}\right|_{K\left(G_{2}\right)}\right) \rightarrow K\left(G_{2}\right)$ can be seen as the universal family of jumping lines of $\mathcal{C}$.

\section{THEOREM 6.5}

Let $(X, \mathcal{E})$ be as in Setup 4.1, and assume that $\mathcal{E}$ is indecomposable. Then the following are equivalent:

(1) $\mathbb{P}(\mathcal{E})$ admits an unsplit dominating family $\mathcal{M}^{\prime}$ of rational curves of positive $H$-degree;

(2) $\mathbb{P}(\mathcal{E})$ has a second contraction which is a $\mathbb{P}^{1}$-bundle;

(3) $(X, \mathcal{E})$ is one of the following;

(a) $\left(\mathbb{P}^{2}, T_{\mathbb{P}^{2}}\right)$;

(b) $\left(\mathbb{P}^{3}, \mathcal{N}\right)$, with $\mathcal{N}$ a null-correlation bundle;

(c) $\left(\mathbb{Q}^{3}, \mathcal{S}\right)$ with $\mathcal{S}$ the restriction of a spinor bundle;

(d) $\left(\mathbb{Q}^{5}, \mathcal{C}\right)$ with $\mathcal{C}$ a Cayley bundle;

(e) $\left(K\left(G_{2}\right), \mathcal{Q}\right)$, with $\mathcal{Q}$ the restriction of the universal quotient bundle.

\section{Proof}

$(1) \Rightarrow(2)$. By Remark 5.6 the dimension of every irreducible component of Chlocus $\left(\mathcal{M}^{\prime}\right)_{y}$ is one, for every $y \in \mathbb{P}(\mathcal{E})$. By [BCD, Proposition 1], this implies that the quotient of $\mathbb{P}(\mathcal{E})$ by the $\mathcal{M}^{\prime}$-equivalence relation is a morphism $\varphi$ : 
$\mathbb{P}(\mathcal{E}) \rightarrow Y$. By Kleiman's criterion $\mathbb{P}(\mathcal{E})$ is a Fano manifold; we conclude by Lemma 6.1 observing that the length of the ray contracted by $\varphi$ is two.

$(2) \Rightarrow(3)$. Assume that $\varphi: \mathbb{P}(\mathcal{E}) \rightarrow Y$ makes $\mathbb{P}(\mathcal{E})$ a $\mathbb{P}^{1}$-bundle over a smooth (Fano) variety $Y$. Let $\mathcal{E}^{\prime}$ be the normalized rank two vector bundle on $Y$ whose projectivization is $\mathbb{P}(\mathcal{E})$, and let $c_{1}^{\prime}$ be its first Chern class. Denote by $L^{\prime}$ a divisor associated with its tautological line bundle, and denote by $H^{\prime}$ the pullback via $\varphi$ of $H_{Y}$, the ample generator of $\operatorname{Pic}(Y)$. Denote $d_{X}:=H_{X}^{n}, d_{Y}:=H_{Y}^{n}$. Finally, take $f$ and $f^{\prime}$ to be fibers of $\pi$ and $\varphi$, respectively, and set $\mu:=H \cdot f^{\prime}, \mu^{\prime}:=H^{\prime} \cdot f$, so that $\tau=\tau(\mathcal{E})=i_{X}-2 / \mu, \tau^{\prime}:=\tau\left(\mathcal{E}^{\prime}\right)=i_{Y}-2 / \mu^{\prime}$.

Using the intersection numbers of $H, L, H^{\prime}$, and $L^{\prime}$ with $f$ and $f^{\prime}$ we may easily write

$$
\left\{\begin{array}{l}
H^{\prime}=-\frac{\mu^{\prime}}{2}\left(c_{1}-\tau\right) H+\mu^{\prime} L, \\
L^{\prime}=\left(-\frac{\mu^{\prime}}{4}\left(c_{1}-\tau\right)\left(c_{1}^{\prime}-\tau^{\prime}\right)+\frac{1}{\mu}\right) H+\frac{\mu^{\prime}}{2}\left(c_{1}^{\prime}-\tau^{\prime}\right) L .
\end{array}\right.
$$

Since $\{H, L\}$ and $\left\{H^{\prime}, L^{\prime}\right\}$ are $\mathbb{Z}$-bases of $\operatorname{Pic}(\mathbb{P}(\mathcal{E}))$ it follows that the determinant of the matrix of base change, which is $\mu^{\prime} / \mu$, is \pm 1 ; hence $\mu=\mu^{\prime}$. In particular, we may write $H^{\prime}=\frac{\mu}{2}\left(-K_{\mathrm{rel}}+\tau H\right)$, so that

$$
\frac{d_{Y}}{d_{X}}=\left(\frac{\mu}{2}\right)^{n} \frac{\left(-K_{\mathrm{rel}}+\tau H\right)^{n} \cdot H / \mu}{-K_{\mathrm{rel}} \cdot H^{n} / 2}=\left(\frac{\mu}{2}\right)^{n-1} \frac{\mathrm{im}\left((\tau+i \sqrt{-\Delta})^{n}\right)}{\sqrt{-\Delta}} .
$$

The last equality follows from our computations in Section 4 and the negativity of $\Delta$ obtained by Proposition 4.12. Furthermore, as in Proposition 4.4, we have $n=2,3$, or 5 and $\sqrt{-\Delta}=\tau \tan (\pi /(n+1))$. Operating in the expression above we get

$$
\frac{d_{Y}}{d_{X}}=\left(\frac{\tau \mu}{2 \cos (\pi /(n+1))}\right)^{n-1}
$$

and this, together with the equation obtained exchanging the roles of $X$ and $Y$, provides

$$
\left(i_{X} \mu-2\right)\left(i_{Y} \mu-2\right)=\tau \tau^{\prime} \mu^{2}= \begin{cases}1 & \text { if } n=2, \\ 2 & \text { if } n=3 \\ 3 & \text { if } n=5 .\end{cases}
$$

From this data we may easily obtain (up to exchanging $X$ and $Y$ ) Table 1 .

Since the tangent bundle of $\mathbb{P}^{2}$, the null-correlation bundle on $\mathbb{P}^{3}$, and the Cayley bundle on $\mathbb{Q}^{5}$ are determined, among stable bundles, by their Chern classes (cf. [H, 8.1], [OSS, Lemma 4.3.2], [O]) and we know that $\mathcal{E}$ is stable by Theorem 6.3 the implication follows.

Table 1

\begin{tabular}{|l|l|l|l|l|l|l|r|l|}
\hline$n$ & $i_{X}$ & $i_{Y}$ & $d$ & $\mu$ & $\tau$ & $\Delta$ & $c_{1}$ & $c_{2}$ \\
\hline 2 & 3 & 3 & 1 & 1 & 1 & -3 & -1 & 1 \\
\hline 3 & 4 & 3 & 1 & 1 & 2 & -4 & 0 & 1 \\
\hline 5 & 5 & 3 & 1 & 1 & 3 & -3 & -1 & 1 \\
\hline
\end{tabular}


$(3) \Rightarrow(1)$. The family of the fibers of the second contraction of $\mathbb{P}(\mathcal{E})$ is unsplit, dominating, and has positive $H$-degree.

\section{COROLLARY 6.6}

Let $(X, \mathcal{E})$ be as in Setup 4.1, and assume that $\mathcal{E}$ is Fano and indecomposable. Then the following are equivalent:

(1) $(X, \mathcal{E})$ is as in Theorem 6.5(3),

(2) $i_{X}-c_{1} \equiv 0(\bmod 2)$,

(3) $\tau<i_{X}-1$.

Proof

$(1) \Rightarrow(2)$ is a direct computation. From (2) it follows that $-K_{\mathbb{P}(\mathcal{E})} \cdot C=2 L \cdot C+$ $\left(i_{X}-c_{1}\right) H \cdot C$ is even, and we conclude (3) by Lemma 6.1 . By Lemma 6.1 again we have that, if (3) holds, then $\tau<i_{X}-1 \leq i_{X}-1 / H \cdot C$. This implies that $\mathbb{P}(\mathcal{E})$ has a second $\mathbb{P}^{1}$-bundle structure, and we get (1) by Theorem 6.5 .

As a consequence we get the following classification of uniform rank two vector bundles on Fano manifolds.

\section{COROLLARY 6.7}

Let $(X, \mathcal{E})$ be as in Setup 4.1, and let $\mathcal{M}$ be a covering unsplit family of rational curves on $X$ such that $\mathcal{M}_{x}$ is irreducible for a general $x \in X$. If $\mathcal{E}$ is indecomposable and uniform with respect to $\mathcal{M}$, then $(X, \mathcal{E})$ is either $\left(\mathbb{P}^{2}, T_{\mathbb{P}^{2}}\right),\left(\mathbb{Q}^{3}, \mathcal{S}\right)$, or $\left(K\left(G_{2}\right), \mathcal{Q}\right)$.

\section{Proof}

Consider the family of minimal sections $\widetilde{\mathcal{M}}$ of $\mathbb{P}(\mathcal{E})$ over $\mathcal{M}$. By Theorem 6.5 either $\operatorname{Locus}(\widetilde{\mathcal{M}})$ is a divisor or $(X, \mathcal{E})$ is one of the pairs listed there. Moreover, checking uniformity in the classification, we get that in the second case $(X, \mathcal{E})$ is $\left(\mathbb{P}^{2}, T_{\mathbb{P}^{2}}\right),\left(\mathbb{Q}^{3}, \mathcal{S}\right)$, or $\left(K\left(G_{2}\right), \mathcal{Q}\right)$.

In particular, it suffices to show that $\mathcal{E}$ splits whenever $\operatorname{Locus}(\widetilde{\mathcal{M}})$ is a divisor. If this is the case, the irreducibility of $\mathcal{M}_{x}$ implies that $\operatorname{Locus}(\widetilde{\mathcal{M}})$ is a unisecant divisor (cf. Remark 3.7), determined by an injection $s: \mathcal{O}(b) \rightarrow \mathcal{E}$. But the general element of $\mathcal{M}$ does not meet the set of zeros of $s$; hence by construction $b=$ $\left(c_{1}+\tau(\ell)\right) / 2$. Note that we may assume that $\tau(\ell)>0$, by [AW, Proposition 1.2]; then we may conclude by Corollary 3.3 .

\section{REMARK 6.8}

Recently, using similar techniques, Watanabe has shown (cf. [Wa]) that the only $\mathbb{P}^{1}$-bundles over Fano manifolds of Picard number one admitting a second smooth fibration of relative dimension one are those listed in our theorem. In his proof no assumption on $b_{4}$ is needed. 


\section{Applications to Hartshorne's conjecture}

Throughout this section we will denote by $Y \subset \mathbb{P}^{n}$, with $n \geq 8$, a codimension two smooth subvariety. By the Barth-Larsen theorem (see, for instance, [L, II, Theorem 3.2.1]), setting $H_{\mathbb{P}^{n}}:=\mathcal{O}_{\mathbb{P}^{n}}(1)$ we obtain that $\operatorname{Pic}(Y) \simeq \mathbb{Z}\left\langle\left(H_{\mathbb{P}^{n}}\right)_{\mid Y}\right\rangle$ and $H^{4}(Y) \simeq \mathbb{Z}\left\langle\left(H_{\mathbb{P}^{n}}\right)_{\mid Y}^{2}\right\rangle$. Let $\mathcal{N}$ be the normal bundle of $Y$ in $\mathbb{P}^{n}$, and let $\mathcal{E}$ be the bundle on $\mathbb{P}^{n}$ obtained from $Y$ via the Hartshorne-Serre correspondence, so that $Y \subset \mathbb{P}^{n}$ appears as the set of zeros of a section $s \in H^{0}\left(\mathbb{P}^{n}, \mathcal{E}\right)$ and $\left.\mathcal{E}\right|_{Y} \simeq \mathcal{N}$.

We will apply the techniques previously developed to the pair $\left(\mathbb{P}^{n}, \mathcal{E}\right)$ (and also to $(Y, \mathcal{N})$, although $Y$ is not necessarily Fano; see Remark 7.3 below). As usual, we will denote by $c_{1}$ and $c_{2}$ the integers determining the first and second Chern classes of $\mathcal{E}$ ( or of $\mathcal{N}$ ), and by $\Delta:=c_{1}^{2}-4 c_{2}$ we denote the discriminant of $\mathcal{E}$. Note that $\mathcal{E}$ is not normalized: in fact, by adjunction and the Kobayashi-Ochiai theorem $c_{1}=(n+1)-i_{Y} \geq 2$.

Set $\tau:=\tau(\mathcal{E}), \rho:=\rho(\mathcal{E})$, denote by $\beta$ the minimum integer such that $\mathcal{E}(\beta)$ has sections, and denote by $\tau_{Y}, \rho_{Y}$, and $\beta_{Y}$ the corresponding invariants of $\mathcal{N}$.

\section{LEMMA 7.1}

Assume that $\Delta \geq 0$. Then $\rho \geq \rho_{Y}$.

Proof

We begin by taking $\ell$ to be a 1 -secant line to $Y$. The restriction $s_{\mid \ell}$ vanishes precisely at one point, so that we have an injection of bundles

$$
0 \rightarrow \mathcal{O}_{\ell}(1) \longrightarrow \mathcal{E}_{\mid \ell}
$$

whose cokernel $\mathcal{E}_{\mid \ell} \rightarrow \mathcal{O}_{\ell}\left(c_{1}-1\right)$ provides a section $\tilde{\ell}$ of $\mathbb{P}(\mathcal{E})$ over $\ell$ verifying $\tau(\tilde{\ell})=-c_{1}+2 \geq 0$. Since $\ell$ is movable and $c_{1}-1 \geq 1$, then $\tilde{\ell}$ is movable and we may assert that $\rho \geq-c_{1}+2$.

Consider a nonzero section $t \in H^{0}\left(\mathbb{P}^{n}, \mathcal{E}(\beta)\right)$; by Proposition 3.5 and Remark 3.6, $\Delta \geq 0$ implies that $\rho=2 \beta+c_{1} \leq 0$. Assume that $\rho<\rho_{Y}$; then $t$ must vanish on $Y$. It follows that

$$
c_{2}(\mathcal{E}(\beta)) \geq[Y]=c_{2}(\mathcal{E})=c_{2}\left(\mathcal{E}\left(-c_{1}\right)\right) .
$$

Since $\beta \leq-c_{1} / 2$ we may then say that $c_{2}(\mathcal{E}(\beta)) \geq c_{2}\left(\mathcal{E}\left(-c_{1}\right)\right)$ implies that $\beta \leq$ $-c_{1}$, contradicting $\rho \geq-c_{1}+2$.

\section{COROLLARY 7.2}

With the same notation as above, assume that $c_{2}\left(\mathcal{N}\left(\beta_{Y}\right)\right)=0$; then $\mathcal{E}$ is decomposable.

\section{Proof}

If $c_{2}\left(\mathcal{N}\left(\beta_{Y}\right)\right)=0$, then $c_{2}\left(\mathcal{E}\left(\beta_{Y}\right)\right)=0$ and we may say that $\Delta \geq 0$; in particular, $\mathcal{E}$ is not stable and $\beta \leq-c_{1} / 2$. Since, moreover, $c_{2}(\mathcal{E}(\beta)) \geq 0$, it follows that $\beta \leq \beta_{Y}$. On the other hand, Lemma 7.1 tells us that $\beta=\left(\rho-c_{1}\right) / 2 \geq\left(\rho_{Y}-c_{1}\right) / 2 \geq \beta_{Y}$, so that $\beta=\beta_{Y}$ and so $c_{2}(\mathcal{E}(\beta))=0$. It follows that $\mathcal{E}$ splits. 


\section{REMARK 7.3}

On a Fano manifold of Picard number one the splitting of a vector bundle $\mathcal{E}$ reduces to $c_{2}(\mathcal{E}(\beta))=0$ via Kodaira vanishing; Corollary 7.2 shows that the same holds for the pair $(Y, \mathcal{N})$. Since this was the only use of assuming $X$ to be Fano in Section 4, this corollary implies, in particular, that the bounds obtained in Section 4 work for the pair $(Y, \mathcal{N})$ as well.

\section{REMARK 7.4}

Let us observe that, being globally generated, $\mathcal{N}(-1)$ is nef; hence $\tau_{Y} \leq c_{1}-2$. (It is well-known that, under our assumptions, $\mathcal{N}(-1)$ is ample, so that the inequality is strict, in fact.)

Let us assume that $\Delta>0$. In [Ho, Corollary 7.3] it is shown that if $\mathcal{E}$ is indecomposable, then $c_{1}>2(n+\sqrt{n+1})$. One of the ingredients of the proof is the fact that $c_{2}(\mathcal{E}(\beta)) \geq n+2$; joining this with our results of Section 4 we are able to find a lower bound for $c_{1}$ of order $3 / 2$.

\section{PROPOSITION 7.5}

If $\Delta>0$ and $c_{1} \leq \sqrt{\left(n^{2}-4\right)(n-3)}+\sqrt{\Delta}+3$, then $\mathcal{E}$ is decomposable.

Proof

By Lemma 4.6 we know that $\rho_{Y}^{2} \leq \Delta+2 \varepsilon \sqrt{\Delta}+\varepsilon^{2}$; hence

$$
\varepsilon^{2}+2 \varepsilon \sqrt{\Delta}-4 c_{2}\left(\mathcal{N}\left(\beta_{Y}\right)\right) \geq 0
$$

so we must have $\varepsilon \geq-\sqrt{\Delta}+\sqrt{\Delta+4 c_{2}\left(\mathcal{N}\left(\beta_{Y}\right)\right)}$. We can rewrite this as

$$
\varepsilon \geq \frac{4 c_{2}\left(\mathcal{N}\left(\beta_{Y}\right)\right)}{\sqrt{\Delta}+\sqrt{\Delta+4 c_{2}\left(\mathcal{N}\left(\beta_{Y}\right)\right)}} .
$$

We have the following bounds:

$$
\varepsilon> \begin{cases}\varepsilon_{1}=\sqrt{c_{2}\left(\mathcal{N}\left(\beta_{Y}\right)\right)} & \text { if } \Delta \leq c_{2}\left(\mathcal{N}\left(\beta_{Y}\right)\right), \\ \varepsilon_{2}=\frac{c_{2}\left(\mathcal{N}\left(\beta_{Y}\right)\right)}{\sqrt{\Delta}} & \text { if } \Delta \geq c_{2}\left(\mathcal{N}\left(\beta_{Y}\right)\right) .\end{cases}
$$

Writing $\varepsilon$ as

$$
\varepsilon=\frac{2 \sqrt{\Delta}}{\left(1+\frac{2 \sqrt{\Delta}}{\tau_{Y}-\sqrt{\Delta}}\right)^{n-2}-1}
$$

we see, taking different terms of the binomial expansion of the denominator, that

$$
\begin{aligned}
& \varepsilon<\delta_{1}=\frac{2 \sqrt{\Delta}}{(n-2)\left(\frac{2 \sqrt{\Delta}}{\left(\tau_{Y}-\sqrt{\Delta}\right)}\right)}=\frac{\tau_{Y}-\sqrt{\Delta}}{n-2}, \\
& \varepsilon<\delta_{2}=\frac{2 \sqrt{\Delta}}{\frac{(n-2)(n-3)}{2}\left(\frac{4 \Delta}{(\tau-\sqrt{\Delta})^{2}}\right)}=\frac{\left(\tau_{Y}-\sqrt{\Delta}\right)^{2}}{(n-2)(n-3) \sqrt{\Delta}} .
\end{aligned}
$$


Now let us write the conditions $\delta_{i}>\varepsilon_{i}$ and Remark 7.4:

$$
\begin{aligned}
& c_{1}-2>\tau_{Y}>\sqrt{\Delta}+(n-2) \sqrt{c_{2}\left(\mathcal{N}\left(\beta_{Y}\right)\right)}, \\
& c_{1}-2>\tau_{Y}>\sqrt{\Delta}+\sqrt{(n-2)(n-3) c_{2}\left(\mathcal{N}\left(\beta_{Y}\right)\right)} .
\end{aligned}
$$

Now we observe that, by Lemma 7.1 we have $\beta_{Y} \leq \beta$; hence $c_{2}\left(\mathcal{N}\left(\beta_{Y}\right)\right) \geq$ $c_{2}(\mathcal{E}(\beta))$, and we use [Ho, Proposition 6.3] to get $c_{2}(\mathcal{E}(\beta)) \geq n+2$ and conclude.

Finally let us consider separately the case in which $Y$ is a numerical complete intersection of type $(a, b) \in \mathbb{Z}^{2}, a \leq b$, that is, when $\Delta=(b-a)^{2} \geq 0$.

\section{REMARK 7.6}

Observe that, by Corollary 7.2, a numerical complete intersection with $\beta_{Y}=-a$ or $-b$ is a complete intersection.

\section{COROLLARY 7.7}

Assume that $Y$ is a numerical complete intersection of type $(a, b)$ with $a \leq b$. If $(n-3)(b-1)>(a-2)(a-1)$, then $Y$ is a complete intersection.

\section{Proof}

We will show that $\mathcal{E}$ is decomposable. By Remark 7.4 we have $\tau_{Y}<c_{1}-2=$ $a+b-2$. Note that, by Proposition 3.5, Remark 3.6, and Remark 4.5, $\mathcal{E}$ splits if and only if $\rho=2 \beta+c_{1}=-\sqrt{\Delta}$. Since $\beta \in \mathbb{Z}$, using Lemma 4.6 , this is equivalent to $\varepsilon<2$.

In the case $\Delta=0$ this gives $\tau_{Y}<2(n-2)$, which is satisfied in our assumptions. In the case $\Delta>0$ we have

$$
\varepsilon=\frac{2 \sqrt{\Delta}\left(\tau_{Y}-\sqrt{\Delta}\right)^{n-2}}{\left(\tau_{Y}+\sqrt{\Delta}\right)^{n-2}-\left(\tau_{Y}-\sqrt{\Delta}\right)^{n-2}}<\frac{2 \sqrt{\Delta}\left(c_{1}-2-\sqrt{\Delta}\right)^{n-2}}{\left(c_{1}-2+\sqrt{\Delta}\right)^{n-2}-\left(c_{1}-2-\sqrt{\Delta}\right)^{n-2}} .
$$

It follows that

$$
\varepsilon<\frac{2(b-a)(a-1)^{n-2}}{(b-1)^{n-2}-(a-1)^{n-2}} .
$$

The condition $\varepsilon<2$ holds trivially when $a=1$; hence we may assume that $b>$ $a>1$ and a sufficient condition for the splitting is

$$
1>\frac{(b-a)(a-1)^{n-2}}{(b-1)^{n-2}-(a-1)^{n-2}}=(a-1) \frac{\left(\frac{b-1}{a-1}\right)-1}{\left(\frac{b-1}{a-1}\right)^{n-2}-1} ;
$$

that is,

$$
\frac{\left(\frac{b-1}{a-1}\right)^{n-2}-1}{\left(\frac{b-1}{a-1}\right)-1}>(a-1) .
$$

In particular, since $(b-1) /(a-1)>1$, this condition holds whenever $1+(n-$ $3)(b-1) /(a-1)>(a-1)$, that is $(n-3)(b-1)>(a-2)(a-1)$. 


\section{REMARK 7.8}

Corollary 7.7 improves the known results about Hartshorne's conjecture for codimension two numerical complete intersections (cf. [EF, Corollary 2.3]).

Acknowledgments. The authors would like to thank E. Ballico for interesting discussions and suggestions, the Korea Institute for Advanced Study (KIAS) for hospitality during part of the preparation of this paper, and C. Casagrande for pointing out a mistake in a previous version of the paper. They would also like to thank the anonymous referees for helpful and constructive comments.

\section{References}

[APW] V. Ancona, T. Peternell, and J. Wiśniewski, Fano bundles and splitting theorems on projective spaces and quadrics, Pacific J. Math. 163 (1994), 17-42. MR 1256175.

[AW] M. Andreatta and J. Wiśniewski, On manifolds whose tangent bundle contains an ample subbundle, Invent. Math. 146 (2001), 209-217. MR 1859022. DOI 10.1007/PL00005808.

[B] E. Ballico, A splitting criterion for rank 2 vector bundles on $\mathbf{P}^{n}$, Pacific J. Math. 169 (1995), 51-52. MR 1346245.

[Bt] W. Barth, Some properties of stable rank-2 vector bundles on $\mathbb{P}_{n}$, Math. Ann. 226 (1977), 125-150. MR 0429896.

[Ba] T. Bauer, Seshadri constants on algebraic surfaces, Math. Ann. 313 (1999), 547-583. MR 1678549. DOI 10.1007/s002080050272.

[BdS] I. Biswas and J. P. dos Santos, On the vector bundles over rationally connected varieties, C. R. Math. Acad. Sci. Paris 347 (2009), 1173-1176. MR 2566998. DOI 10.1016/j.crma.2009.09.006.

[BCD] L. Bonavero, C. Casagrande, and S. Druel, On covering and quasi-unsplit families of rational curves, J. Eur. Math. Soc. (JEMS) 9 (2007), 45-57. MR 2283102. DOI 10.4171/JEMS/71.

[BDPP] S. Boucksom, J.-P. Demailly, M. Păun, and T. Peternell, The pseudo-effective cone of a compact Kähler manifold and varieties of negative Kodaira dimension, preprint, arXiv:math/0405285v1 [math.AG]

[Ca] J. S. Calcut, Rationality and the tangent function, preprint.

[D] O. Debarre, Higher-Dimensional Algebraic Geometry, Universitext, Springer, New York 2001. MR 1841091.

[EF] Ph. Ellia and D. Franco, On codimension two subvarieties of $\mathbf{P}^{5}$ and $\mathbf{P}^{6}$, J. Algebraic Geom. 11 (2002), 513-533. MR 1894936.

DOI 10.1090/S1056-3911-02-00320-X.

[Fl] H. Flenner, Restrictions of semistable bundles on projective varieties, Comment. Math. Helv. 59 (1984), 635-650. MR 0780080.

DOI $10.1007 / \mathrm{BF} 02566370$. 
[FHS] O. Forster, A. Hirschowitz, and M. Schneider, "Type de scindage généralisé pour les fibrés stables" in Vector Bundles and Differential Equations (Nice, 1979), Progr. Math. 7, Birkhäuser, Boston, 1980, 65-81. MR 0589221.

[F] T. Fujita, Classification Theories of Polarized Varieties, London Math. Soc. Lecture Ser. 155, Cambridge Univ. Press, Cambridge, 1990. MR 1162108. DOI 10.1017/CBO9780511662638.

[GS] H. Grauert and M. Schneider, Komplexe Unterräume und holomorphe Vektorraumbündel vom Rang zwei, Math. Ann. 230 (1977), 75-90. MR 0481134.

[Ha1] R. Hartshorne, Varieties of small codimension in projective space, Bull. Amer. Math. Soc. 80 (1974), 1017-1032. MR 0384816.

[Ha2] , Algebraic Geometry, Grad. Texts in Math. 52, Springer, New York, 1977. MR 0463157.

[Hi] A. Hirschowitz, Sur la restriction des faisceaux semi-stables, Ann. Sci. École Norm. Sup. (4) 14 (1981), 199-207. MR 0631750.

[Ho] A. Holme, Codimension 2 subvarieties of projective space, Manuscripta Math. 65 (1989), 427-446. MR 1019701. DOI 10.1007/BF01172790.

[HM] G. Horrocks and D. Mumford, A rank 2 vector bundle on $\mathbf{P}^{4}$ with 15,000 symmetries, Topology 12 (1973), 63-81. MR 0382279.

[H] K. Hulek, Stable rank-2 vector bundles on $\mathbf{P}_{2}$ with $c_{1}$ odd, Math. Ann. 242 (1979), 241-266. MR 0545217. DOI 10.1007/BF01420729.

[HL] D. Huybrechts and M. Lehn, The Geometry of the Moduli Spaces of Sheaves, 2nd ed., Cambridge Math. Lib., Cambridge Univ. Press, Cambridge, 2010. MR 2665168. DOI 10.1017/CBO9780511711985.

[K] J. Kollár, Rational curves on algebraic varieties, Ergeb. Math. Grenzgeb. (3) 32, Springer, Berlin, 1996. MR 1440180.

[KMM] J. Kollár, Y. Miyaoka, and S. Mori, Rational connectedness and boundedness of Fano manifolds, J. Differential Geom. 36 (1992), 765-779. MR 1189503.

[L] R. Lazarsfeld, Positivity in Algebraic Geometry, II: Positivity for Vector Bundles, and Multiplier Ideals, Ergeb. Math. Grenzgeb. (3) 49, Springer, Berlin, 2004. MR 2095472.

[MR1] V. B. Mehta and A. Ramanathan, Semistable sheaves on projective varieties and their restriction to curves, Math. Ann. 258 (1981/82), 213-224. MR 0649194. DOI 10.1007/BF01450677.

[MR2] - Restriction of stable sheaves and representations of the fundamental group, Invent. Math. 77 (1984), 163-172. MR 0751136. DOI 10.1007/BF01389140.

[M] S. Mukai, Biregular classification of Fano 3-folds and Fano manifolds of coindex 3, Proc. Nat. Acad. Sci. U.S.A. 86 (1989), 3000-3002.

MR 0995400. DOI 10.1073/pnas.86.9.3000. 
[MOS1] R. Muñoz, G. Occhetta, and L. E. Solá Conde, Uniform vector bundles on Fano manifolds and applications, J. Reine Angew. Math. 664 (2012), 141-162. MR 2980134.

[MOS2] _ A classification theorem on Fano bundles, to appear in Ann. Inst. Fourier, preprint, arXiv:1204.4793v1 [math.AG].

[N] M. Nagata, On the 14th problem of Hilbert, Amer. J. Math. 81 (1959), 766-783. MR 0105409.

[Ni] I. Niven, Irrational Numbers, Carus Math. Monogr. 11, Math. Assoc. America, distributed by Wiley, New York, 1956. MR 0080123.

[OSS] C. Okonek, M. Schneider, and H. Spindler, Vector Bundles on Complex Projective Spaces, Progr. Math. 3, Birkhäuser, Boston, 1980. MR 0561910.

[O] Ottaviani, G., On Cayley bundles on the five-dimensional quadric, Boll. Un. Mat. Ital. A (7) 4, no. 1, 87-100 (1990). MR 1047517.

[S] V. G. Sarkisov, On conic bundle structures (in Russian), Izv. Akad. Nauk SSSR Ser. Mat. 46 (1982), 371-408. MR 0651652.

[Su] H. Sumihiro, Determinantal varieties associated to rank two vector bundles on projective spaces and splitting theorems, Hiroshima Math. J. 29 (1999), 371-434. MR 1704256.

[SW1] M. Szurek and J. Wiśniewski, Fano bundles over $\mathbf{P}^{3}$ and $Q_{3}$, Pacific J. Math. 141 (1990), 197-208. MR 1028270.

[SW2] On Fano manifolds, which are $\mathbf{P}^{k}$-bundles over $\mathbf{P}^{2}$, Nagoya Math. J. 120 (1990), 89-101. MR 1086572.

[Wa] Watanabe, K., $\mathbb{P}^{1}$-bundles over projective manifolds of Picard number one which admit another smooth morphism of relative dimension one, arXiv:1201.3558v3 [math.AG].

[W] Wiśniewski. J., On contractions of extremal rays of Fano manifolds, J. Reine Angew. Math. 417 (1991), 141-157. MR 1103910. DOI 10.1515/crll.1991.417.141.

Muñoz: Departamento de Matemática Aplicada, ESCET, Universidad Rey Juan Carlos, 28933-Móstoles, Madrid, Spain; roberto.munoz@urjc.es

Occhetta: Dipartimento di Matematica, Università di Trento, via Sommarive 14 I-38123 Povo (TN), Italy; gianluca.occhetta@unitn.it

Solá Conde: Departamento de Matemática Aplicada, ESCET, Universidad Rey Juan Carlos, 28933 Móstoles, Madrid, Spain. Center for Biomedical Technology, Universidad Politécnica de Madrid, Campus Montegancedo, 28223 Pozuelo de Alarcón, Madrid, Spain; luis.sola@urjc.es 